\title{
SUPERCONVERGENCE ANALYSIS OF LOW ORDER NONCONFORMING MIXED FINITE ELEMENT METHODS FOR TIME-DEPENDENT NAVIER-STOKES EQUATIONS*
}

\author{
Huaijun Yang \\ School of Mathematics, Zhengzhou University of Aeronautics, Zhengzhou 450046, China \\ Email:yhjfhw@163.com \\ Dongyang $\mathrm{Shi}^{1)}$ and Qian Liu \\ School of Mathematics and Statistics, Zhengzhou University, Zhengzhou 450001, China \\ Email: shi_dy@zzu.edu.cn, zzshidy@126.com,lylq1990@163.com
}

\begin{abstract}
In this paper, the superconvergence properties of the time-dependent Navier-Stokes equations are investigated by a low order nonconforming mixed finite element method (MFEM). In terms of the integral identity technique, the superclose error estimates for both the velocity in broken $H^{1}$-norm and the pressure in $L^{2}$-norm are first obtained, which play a key role to bound the numerical solution in $L^{\infty}$-norm. Then the corresponding global superconvergence results are derived through a suitable interpolation postprocessing approach. Finally, some numerical results are provided to demonstrated the theoretical analysis.
\end{abstract}

Mathematics subject classification: 65N38, 65N30, 65M60, 65M12.

Key words: Navier-Stokes equations, Nonconforming MFEM, Supercloseness and superconvergence.

\section{Introduction}

In this paper, we focus on the following time-dependent Navier-Stokes equations in 2D:

$$
\begin{array}{ll}
\boldsymbol{u}_{t}-\nu \Delta \boldsymbol{u}+(\boldsymbol{u} \cdot \nabla) \boldsymbol{u}+\nabla p=\boldsymbol{f}, & (\boldsymbol{x}, t) \in \Omega \times(0, T], \\
\nabla \cdot \boldsymbol{u}=0, & (\boldsymbol{x}, t) \in \Omega \times(0, T], \\
\boldsymbol{u}(\boldsymbol{x}, t)=0, & (\boldsymbol{x}, t) \in \partial \Omega \times(0, T], \\
\boldsymbol{u}(\boldsymbol{x}, 0)=\boldsymbol{u}_{0}(\boldsymbol{x}), & \boldsymbol{x} \in \Omega,
\end{array}
$$

where $\Omega \subset \mathbb{R}^{2}$ is a rectangular domain with boundary $\partial \Omega$ and $\boldsymbol{x}=\left(x_{1}, x_{2}\right) . \quad \boldsymbol{u}=\left(u_{1}, u_{2}\right)$ represents the velocity vector, $p$ the pressure, $f=\left(f_{1}, f_{2}\right)$ the body force, $\nu=1 / R e$ the viscosity coefficient and $R e$ is the Reynolds number.

It is well known that the incompressible Navier-Stokes equations are of great importance both in mathematics and fluid mechanics. There have been a large number of works concentrated on the numerical solutions of Navier-Stokes equations. We refer the readers to monographs $[1,2]$ for the theoretical and numerical analysis, [3-6] for finite difference methods, [7-24] for FEMs, [25-27] for characteristics FEMs, [28, 29] for discontinuous Galerkin method. More

\footnotetext{
* Received December 2, 2018 / Revised version received May 12, 2019 / Accepted July 17, 2019 /

Published online August 16, 2019 /

1) Corresponding author
} 
precisely, ax fast finite difference method was proposed in [4] based on the vorticity streamfunction formulation. A backward Euler fully-discrete penalty FEM was presented in [7] and an optimal error estimate was provided when the corresponding parameters were sufficiently small. Through the spatial discretization by finite element approximation and the time discrtization by the semi-implicit scheme, a fully-discrete stabilized FEM was studied in [8]. In addition, a stabilized FEM was considered by use of the local polynomial pressure projection with the lowest equal-order elements in [9]. The two-level finite element Galerkin method was employed to deduce the corresponding optimal error estimates in [10] and [11], respectively. Moreover, a class of nonconforming rectangular elements were used in [16] and an optimal estimate was obtained. Two kinds second order nonconforming mixed FEMs were developed and optimal error estimates were derived in [22] and [23], respectively. In [27], the unconditional stability and convergence of characteristics type method was studied and an optimal error estimate was achieved.

As far as we know, all of the above works are concerned with convergence analysis and optimal error estimates. Recently, the superconvergnece analysis was researched with nonconforming mixed FEM $\left(\mathrm{CNR} Q_{1}+Q_{0}\right.$, see the Section 2 for the definition) for the stationary Navier-Stokes equations and time-dependent Navier-Stokes equations in [30] and [31], respectively. However, only the error estimate for the spatial semi-discrete scheme was considered in [31] and the error estimate is not valid when $t \rightarrow 0$.

In this paper, we will focus on the superconvergence analysis for (1.1)-(1.4) by a linearized fully-discrete scheme, in which the spatial discretization approximated by the low order $\operatorname{CNR} Q_{1}$ element (cf. [32,33]) for the velocity, and the piecewise constant for the pressure and the time discretization approximated by the semi-implicit Euler scheme. It should be mentioned that the factor $1 / t$ required in [31] is removed in our present work, which shows that the error estimates are also valid when $t \rightarrow 0$.

The rest of this paper is organized as follows. In Section 2, we briefly introduce the nonconforming finite element spaces and some lemmas. In Section 3, we discuss the superclose and superconvergence analysis for (1.1)-(1.4). In the last section, we carry out two numerical experiments to confirm the theoretical analysis.

\section{The Finite Element Spaces and Some Lemmas}

We will use the standard notations for the Sobolev space $H^{m}(\Omega), m \geq 0$ (cf. [34]) with their associated norm $\|\cdot\|_{m}$ and seminorm $|\cdot|_{m}$. In the case $m=0$, then $H^{0}(\Omega)=L^{2}(\Omega)$, the norm and inner product are denoted by $\|\cdot\|_{0}$ and $(\cdot, \cdot)$, respectively. We let $L_{0}^{2}(\Omega)$ denote the subspace of $L^{2}(\Omega)$ such that

$$
L_{0}^{2}(\Omega)=\left\{v \in L^{2}(\Omega): \int_{\Omega} v d x_{1} d x_{2}=0\right\} .
$$

In addition, for any Banach space $X$ and $I=[0, T]$, let $L^{p}(I ; X)$ be the space of all measurable function $f: I \rightarrow X$ with the norm

$$
\|f\|_{L^{p}(I ; X)}= \begin{cases}\left(\int_{0}^{T}\|f\|_{X}^{p} d t\right)^{\frac{1}{p}}, & 1 \leq p<\infty \\ \operatorname{esssup}_{t \in I}\|f\|_{X}, & p=\infty\end{cases}
$$


The weak formulation of (1.1)-(1.4) reads as: find $\boldsymbol{u}:[0, T] \rightarrow\left(H_{0}^{1}(\Omega)\right)^{2}$ and $p:[0, T] \rightarrow L_{0}^{2}(\Omega)$, such that

$$
\begin{aligned}
& \left(\boldsymbol{u}_{t}, \boldsymbol{v}\right)+\nu a(\boldsymbol{u}, \boldsymbol{v})+c(\boldsymbol{u} ; \boldsymbol{u}, \boldsymbol{v})-b(p, \boldsymbol{v})=(\boldsymbol{f}, \boldsymbol{v}), \quad \forall \boldsymbol{v} \in\left(H_{0}^{1}(\Omega)\right)^{2}, \\
& b(q, \boldsymbol{u})=0, \quad \forall q \in L_{0}^{2}(\Omega),
\end{aligned}
$$

where

$$
\begin{aligned}
& a(\boldsymbol{u}, \boldsymbol{v})=\int_{\Omega} \nabla \boldsymbol{u}: \nabla \boldsymbol{v} d x_{1} d x_{2}, \quad b(q, \boldsymbol{u})=\int_{\Omega} \nabla \cdot \boldsymbol{u} q d x_{1} d x_{2}, \\
& c(\boldsymbol{u} ; \boldsymbol{u}, \boldsymbol{v})=\int_{\Omega}(\boldsymbol{u} \cdot \nabla) \boldsymbol{u} \cdot \boldsymbol{v} d x_{1} d x_{2} .
\end{aligned}
$$

Let $\mathcal{T}_{h}=\{e\}$ be a uniform rectangular mesh over $\Omega$ with mesh size $h$. For a given element $e \in \mathcal{T}_{h}$, its four nodes are denoted by $a_{i}=\left(x_{1 i}, x_{2 i}\right), i=1,2,3,4$, in the counterclockwise order (see Fig. 2.1). Moreover, $l_{i}=\overline{a_{i} a_{i+1}}(\bmod 4), i=1,2,3,4$, are the four edges of element $e$. Let $\hat{e}=[-1,1]^{2}$ denote the reference element with nodes $\hat{a}_{i}, i=1,2,3,4$. Define the bilinear transformation $\mathcal{F}_{e}: \hat{e} \rightarrow e$ by

$$
x_{1}=\sum_{i=1}^{4} x_{1 i} N_{i}(\xi, \eta), \quad x_{2}=\sum_{i=1}^{4} x_{2 i} N_{i}(\xi, \eta), \quad(\xi, \eta) \in \hat{e},
$$

where $N_{i}(\xi, \eta), i=1,2,3,4$ are the bilinear basis functions, which can be written as

$$
\begin{array}{ll}
N_{1}(\xi, \eta)=\frac{1}{4}(1-\xi)(1-\eta), & N_{2}(\xi, \eta)=\frac{1}{4}(1+\xi)(1-\eta), \\
N_{3}(\xi, \eta)=\frac{1}{4}(1+\xi)(1+\eta), & N_{4}(\xi, \eta)=\frac{1}{4}(1-\xi)(1+\eta) .
\end{array}
$$

For edge $l \subset \partial e$, the edge functional $i_{h} v$ is defined as

$$
\left.i_{h} v\right|_{l}=\frac{1}{|l|} \int_{l} v d s, \quad \forall v \in L^{2}(e) .
$$

Here, we briefly describe the construction of $\mathrm{CNR}_{1}$ element (cf. [32,33]). Since the CNR $Q_{1}$ element is obtained from the nonconforming rotated $Q_{1}\left(\mathrm{NR} Q_{1}\right)$ element (cf. [35]) by imposing a constraint (cf. [36]) on each element. The $\mathrm{NR}_{1}$ element space $R^{h}$ is defined as:

$$
R^{h}=\left\{v \in L^{2}(\Omega):\left.v\right|_{e}=\hat{v} \circ \mathcal{F}_{e}^{-1}, \hat{v} \in \operatorname{span}\left\{1, \xi, \eta, \xi^{2}-\eta^{2}\right\}, v \text { is continuous regarding } i_{h}\right\} .
$$

and the corresponding homogenous space is

$$
R_{0}^{h}=\left\{v \in R^{h}:\left.i_{h} v\right|_{l}=0, \text { if } l \subset \partial \Omega\right\} .
$$

Hence, with the help of the spaces $R^{h}$ and $R_{0}^{h}$, we state the constrained nonconforming rotated $Q_{1}\left(\mathrm{CNR} Q_{1}\right.$ for short) element space $C R^{h}$ and its homogenous space $C R_{0}^{h}$ as:

$$
\begin{aligned}
C R^{h} & =\left\{v \in R^{h}: \frac{1}{\left|l_{1}\right|} \int_{l_{1}} v d s+\frac{1}{\left|l_{3}\right|} \int_{l_{3}} v d s=\frac{1}{\left|l_{2}\right|} \int_{l_{2}} v d s+\frac{1}{\left|l_{4}\right|} \int_{l_{4}} v d s, \forall e \in \mathcal{T}_{h}\right\}, \\
C R_{0}^{h} & =\left\{v \in R_{0}^{h}: \frac{1}{\left|l_{1}\right|} \int_{l_{1}} v d s+\frac{1}{\left|l_{3}\right|} \int_{l_{3}} v d s=\frac{1}{\left|l_{2}\right|} \int_{l_{2}} v d s+\frac{1}{\left|l_{4}\right|} \int_{l_{4}} v d s, \forall e \in \mathcal{T}_{h}\right\} .
\end{aligned}
$$


Let $N_{i}^{V}$ denote the number of interior nodes. It has been proven in $[32,36]$ that $\operatorname{dim}\left(C R_{0}^{h}\right)=N_{i}^{V}$. For completeness, we present the basis of $C R_{0}^{h}$ (cf. [32]). Firstly, on the reference element $\hat{e}$, define

$$
\begin{aligned}
\hat{\phi}_{1} & =\frac{1}{4}(1-\xi-\eta), & \hat{\phi}_{2} & =\frac{1}{4}(1+\xi-\eta), \\
\hat{\phi}_{3} & =\frac{1}{4}(1+\xi+\eta), & \hat{\phi}_{4} & =\frac{1}{4}(1-\xi+\eta),
\end{aligned}
$$

which are associated with nodes $\hat{a}_{i}, i=1,2,3,4$, of $\hat{e}$. In particular, it holds that

$$
\int_{\hat{l}_{1}} \hat{\phi}_{i} d \hat{s}+\int_{\hat{l}_{3}} \hat{\phi}_{i} d \hat{s}=\int_{\hat{l}_{2}} \hat{\phi}_{i} d \hat{s}+\int_{\hat{l}_{4}} \hat{\phi}_{i} d \hat{s}, \quad i=1,2,3,4 .
$$

Secondly, for each interior node $a_{j}, j=1,2, \ldots, N_{i}^{V}$, let $E(j)$ denote the set of elements with the node $a_{j}$ as one of their vertexes. Then we define

$$
\phi_{j}(a)= \begin{cases}\hat{\phi}_{i}\left(\mathcal{F}_{e}^{-1}(a)\right), & a \in e \in E(j), \\ 0, & a \in e \in \mathcal{T}_{h} \backslash E(j),\end{cases}
$$

where the subscript $i$ is determined by $a_{j}=a_{i, e}=\mathcal{F}_{e}\left(\hat{a}_{i}\right)$ with $a_{i, e}, i=1,2,3,4$, the four nodes of element $e$. It is easy to see that $\phi_{j}, j=1, \ldots, N_{i}^{V}$ are linearly independent and that

$$
\operatorname{span}\left\{\phi_{i}, \cdots, \phi_{N_{i}^{V}}\right\} \subset C R_{0}^{h},
$$

therefore, $\left\{\phi_{j}\right\}_{j=1}^{N_{i}^{V}}$ is a basis of $C R_{0}^{h}$.
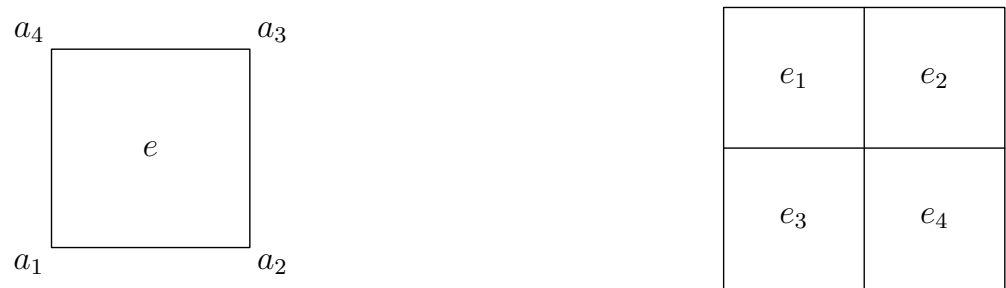

Fig. 2.1. The element $e$ (left) and $\tilde{e}$ (right).

For the velocity, we choose $\boldsymbol{V}_{h}=C R_{0}^{h} \times C R_{0}^{h}$ as finite element space. For the pressure, we assume that the subdivision $\mathcal{T}_{h}$ is obtained from $\mathcal{T}_{2 h}=\{\tilde{e}\}$ by dividing each element of $\mathcal{T}_{2 h}$ into four small congruent rectangles. Let $P_{h}^{\prime}$ consist of piecewise constant functions with respect to $\mathcal{T}_{h}$ and the local basis functions for $P_{h}^{\prime}$ on a $2 \times 2$-patch of $\tilde{e}$ (see Fig. 2.1) are indicated in Fig. 2.2. Then, the finite element space for pressure is defined by $P_{h}^{\prime} \cap L_{0}^{2}(\Omega)$. In what follows, we always assume that $\tilde{e}=\cup_{i=1}^{4} e_{i} \in \mathcal{T}_{2 h}$ with $e_{i} \in \mathcal{T}_{h}(1 \leq i \leq 4)$ (see Fig. 2.1). Thus, $\boldsymbol{V}_{h}$ and $P_{h}$ are described by

$$
\boldsymbol{V}_{h}=C R_{0}^{h} \times C R_{0}^{h}, \quad P_{h}=\left\{p \in L_{0}^{2}(\Omega):\left.p\right|_{\tilde{e}}=\sum_{i=1}^{3} \lambda_{i}^{\tilde{e}} \varphi_{i}^{\tilde{e}}, \forall \tilde{e} \in \mathcal{T}_{2 h}\right\}
$$

It is easy to see that $|\cdot|_{h}=\left\{\sum_{e \in \mathcal{T}_{h}}|\cdot|_{1, e}^{2}\right\}^{1 / 2}$ is a norm on the space $\boldsymbol{V}_{h}$. 
Moreover, let $0=t_{0}<t_{1}<\cdots<t_{N}=T$ be a given uniform partition of the time interval with time step $\tau=T / N$ and $t_{n}=n \tau, n=0,1, \cdots, N$. For a smooth function $u$ defined on $[0, T]$, denote

$$
u^{n}=u\left(t_{n}\right), \quad D_{\tau} u^{n}=\frac{u^{n}-u^{n-1}}{\tau}
$$

\begin{tabular}{|l|l|}
\hline 1 & 1 \\
\hline 1 & 1 \\
\hline \multicolumn{2}{|c|}{$\varphi_{1}^{\tilde{e}}$}
\end{tabular}

\begin{tabular}{|l|l|}
\hline 1 & -1 \\
\hline 1 & -1 \\
\hline \multicolumn{2}{|c|}{$\varphi_{2}^{\tilde{e}}$}
\end{tabular}

\begin{tabular}{|c|c|}
\hline 1 & 1 \\
\hline-1 & -1 \\
\hline \multicolumn{1}{|c|}{$\varphi_{3}^{\tilde{e}}$}
\end{tabular}

Fig. 2.2. Local basis functions of $P_{h}^{\prime}$.

Then, the linearized fully-discrete approximation of (2.1)-(2.2) is: for given $\boldsymbol{U}_{h}^{n-1} \in \boldsymbol{V}_{h}$, find $\left(\boldsymbol{U}_{h}^{n}, P_{h}^{n}\right) \in \boldsymbol{V}_{h} \times P_{h}$, such that

$$
\begin{aligned}
& \left(D_{\tau} \boldsymbol{U}_{h}^{n}, \boldsymbol{v}_{h}\right)+\nu a_{h}\left(\boldsymbol{U}_{h}^{n}, \boldsymbol{v}_{h}\right)+c_{h}\left(\boldsymbol{U}_{h}^{n-1} ; \boldsymbol{U}_{h}^{n}, \boldsymbol{v}_{h}\right)+b_{h}\left(P_{h}^{n}, \boldsymbol{v}_{h}\right)=\left(\boldsymbol{f}^{n}, \boldsymbol{v}_{h}\right), \quad \forall \boldsymbol{v}_{h} \in \boldsymbol{V}_{h}, \\
& b_{h}\left(q_{h}, \boldsymbol{U}_{h}^{n}\right)=0, \quad \forall q_{h} \in P_{h},
\end{aligned}
$$

where

$$
\begin{aligned}
& a_{h}\left(\boldsymbol{U}_{h}^{n}, \boldsymbol{v}_{h}\right)=\left(\nabla \boldsymbol{U}_{h}^{n}, \nabla \boldsymbol{v}_{h}\right)_{h}=\sum_{e} \int_{e} \nabla \boldsymbol{U}_{h}^{n}: \nabla \boldsymbol{v}_{h} d x_{1} d x_{2}, \\
& b_{h}\left(q_{h}, \boldsymbol{U}_{h}^{n}\right)=\left(\nabla \cdot \boldsymbol{U}_{h}^{n}, q_{h}\right)_{h}=\sum_{e} \int_{e} \nabla \cdot \boldsymbol{U}_{h}^{n} q_{h} d x_{1} d x_{2}, \\
& c_{h}\left(\boldsymbol{U}_{h}^{n-1} ; \boldsymbol{U}_{h}^{n}, \boldsymbol{v}_{h}\right)=\left(\left(\boldsymbol{U}_{h}^{n-1} \cdot \nabla\right) \boldsymbol{U}_{h}^{n}, \boldsymbol{v}_{h}\right)_{h}=\sum_{e} \int_{e}\left(\boldsymbol{U}_{h}^{n-1} \cdot \nabla\right) \boldsymbol{U}_{h}^{n} \cdot \boldsymbol{v}_{h} d x_{1} d x_{2} .
\end{aligned}
$$

It has been shown in $[32,33]$ that the nonconforming finite element pair $\left(\boldsymbol{V}_{h}, P_{h}\right)$ satisfies the discrete Babuška-Brezzi condition, i.e., there exists a constant $\beta>0$, such that

$$
\sup _{0 \neq \boldsymbol{v}_{h} \in \boldsymbol{V}_{h}} \frac{\left(q_{h}, \nabla \cdot \boldsymbol{v}_{h}\right)_{h}}{\left\|\boldsymbol{v}_{h}\right\|_{h}} \geq \beta\left\|q_{h}\right\|_{0}, \quad \forall q_{h} \in P_{h} .
$$

From [16,30], the space $\boldsymbol{V}_{h}$ also satisfies the discrete embedding inequality, i.e.,

$$
\left\|\boldsymbol{v}_{h}\right\|_{0,2 k} \leq C(k)\left\|\boldsymbol{v}_{h}\right\|_{h}, \quad \forall \boldsymbol{v}_{h} \in \boldsymbol{V}_{h}, \quad k=1,2, \ldots,
$$

where $C(k)$ is a constant independent of $h$.

Now, we recall some lemmas, which play a key role in the error analysis.

Lemma 2.1 ([33]). Let $e \in \mathcal{T}_{h}$ be a rectangular mesh and $\phi \in H^{3}(e)$. Then when $v$ is a constant on $e$, we have

$$
\left(\left(\phi-\Pi_{h} \phi\right)_{x_{1}}, v\right)_{e}+\left(\left(\phi-\Pi_{h} \phi\right)_{x_{2}}, v\right)_{e} \leq C h^{2}\|\phi\|_{3, e}\|v\|_{0, e},
$$

where $\Pi_{h}$ is the interpolation operator on $\boldsymbol{V}_{h}$. Furthermore, for $\boldsymbol{u} \in\left(H^{3}(\Omega)\right)^{2}$, there holds

$$
\left(\nabla\left(\boldsymbol{u}-\Pi_{h} \boldsymbol{u}\right), \nabla \boldsymbol{v}_{h}\right)_{h} \leq C h^{2}\|\boldsymbol{u}\|_{3}\left\|\boldsymbol{v}_{h}\right\|_{h}, \quad \forall \boldsymbol{v}_{h} \in \boldsymbol{V}_{h}
$$

Here and later, $C$ is a generic positive constant independent of $n, \tau, h$, but may dependent on the different norms of $\boldsymbol{u}$ and $p$. 
Lemma 2.2 ([33]). If $\mathcal{T}_{h}$ is a rectangular mesh, $\boldsymbol{u} \in\left(H^{3}(\Omega)\right)^{2}$ and $p \in H^{2}(\Omega)$, then

$$
\begin{array}{ll}
\left(\nabla \cdot\left(\boldsymbol{u}-\Pi_{h} \boldsymbol{u}\right), q_{h}\right)_{h} \leq C h^{2}\|\boldsymbol{u}\|_{3}\left\|_{q_{h}}\right\|_{0}, \quad \forall q_{h} \in P_{h}, & \\
\left(p-J_{h} p, \nabla \cdot \boldsymbol{v}_{h}\right)_{h} \leq C h^{2}\|p\|_{2}\left\|\boldsymbol{v}_{h}\right\|_{h}, \quad \forall \boldsymbol{v}_{h} \in \boldsymbol{V}_{h}, & \\
\sum_{e} \int_{\partial e}\left(\nu \frac{\partial \boldsymbol{u}}{\partial \boldsymbol{n}}-p \cdot \boldsymbol{n}\right) \boldsymbol{v}_{h} d s \leq C h^{2}\left(\|\boldsymbol{u}\|_{3}+\|p\|_{2}\right)\left\|\boldsymbol{v}_{h}\right\|_{h}, \quad \forall \boldsymbol{v}_{h} \in \boldsymbol{V}_{h} .
\end{array}
$$

Lemma 2.3 ([15]). Let $\tau, D$ and $\left\{a_{n}\right\},\left\{b_{n}\right\},\left\{c_{n}\right\},\left\{d_{n}\right\}$ be nonnegative numbers such that

$$
a_{n}+\tau \sum_{i=0}^{n} b_{i} \leq \tau \sum_{i=0}^{n} d_{i} a_{i}+\tau \sum_{i=0}^{n} c_{i}+D
$$

fora $n \geq 0$. Suppose that $\tau d_{i}<1$ for all $i$. Then

$$
a_{n}+\tau \sum_{i=0}^{n} b_{i} \leq \exp \left(\tau \sum_{i=0}^{n} \frac{d_{i}}{1-\tau d_{i}}\right)\left(\tau \sum_{i=0}^{n} c_{i}+D\right) \text {. }
$$

\section{The Superclose and Superconvergent Error Estimates}

In this section, we will present the main results of our paper.

Theorem 3.1. Let $\left(\boldsymbol{u}^{n}, p^{n}\right)$ and $\left(\boldsymbol{U}_{h}^{n}, P_{h}^{n}\right)$ be the solutions of (2.1)-(2.2) and (2.4)-(2.5) at $t=$ $t_{n}$, respectively. For each $t \in(0, T]$, assume that $\boldsymbol{u}, \boldsymbol{u}_{t} \in\left(L^{\infty}\left(H^{3}(\Omega)\right)\right)^{2}, \boldsymbol{u}_{t t} \in\left(L^{\infty}\left(L^{2}(\Omega)\right)\right)^{2}$, $p \in L^{\infty}\left(H^{2}(\Omega)\right)$ and $p_{t} \in L^{2}\left(L^{2}(\Omega)\right)$, then for any integer number $1 \leq n \leq N$, we have

$$
\left\|\Pi_{h} \boldsymbol{u}^{n}-\boldsymbol{U}_{h}^{n}\right\|_{h}+\tau \sum_{i=1}^{n}\left\|J_{h} p^{i}-P_{h}^{i}\right\|_{0} \leq C\left(h^{2}+\tau\right) .
$$

Proof. Denote

$$
\begin{aligned}
& \boldsymbol{u}^{n}-\boldsymbol{U}_{h}^{n}=\boldsymbol{u}^{n}-\Pi_{h} \boldsymbol{u}^{n}+\Pi_{h} \boldsymbol{u}^{n}-\boldsymbol{U}_{h}^{n}:=\boldsymbol{\rho}^{n}+\boldsymbol{\theta}^{n}, \\
& p^{n}-P_{h}^{n}=p^{n}-J_{h} p^{n}+J_{h} p^{n}-P_{h}^{n}:=\xi^{n}+\eta^{n} .
\end{aligned}
$$

From (2.1)-(2.2) and (2.4)-(2.5), we have the following error equations:

$$
\begin{aligned}
& \left(D_{\tau} \boldsymbol{\theta}^{n}, \boldsymbol{v}_{h}\right)+\nu\left(\nabla \boldsymbol{\theta}^{n}, \nabla \boldsymbol{v}_{h}\right)_{h}-\left(\eta^{n}, \nabla \cdot \boldsymbol{v}_{h}\right)_{h} \\
= & -\left(D_{\tau} \boldsymbol{\rho}^{n}, \boldsymbol{v}_{h}\right)-\nu\left(\nabla \boldsymbol{\rho}^{n}, \nabla \boldsymbol{v}_{h}\right)_{h}+\left(\xi^{n}, \nabla \cdot \boldsymbol{v}_{h}\right)_{h}+\left(\boldsymbol{R}^{n}, \boldsymbol{v}_{h}\right) \\
& -\left(\left(\boldsymbol{u}^{n-1} \cdot \nabla\right) \boldsymbol{u}^{n}-\left(\boldsymbol{U}_{h}^{n-1} \cdot \nabla\right) \boldsymbol{U}_{h}^{n}, \boldsymbol{v}_{h}\right)_{h}+\sum_{e} \int_{\partial e}\left(\nu \frac{\partial \boldsymbol{u}^{n}}{\partial \boldsymbol{n}}-p^{n} \cdot \boldsymbol{n}\right) \boldsymbol{v}_{h} d s, \quad \forall \boldsymbol{v}_{h} \in \boldsymbol{V}_{h}, \\
& \left(\nabla \cdot \boldsymbol{\rho}^{n}, q_{h}\right)_{h}+\left(\nabla \cdot \boldsymbol{\theta}^{n}, q_{h}\right)_{h}, \quad \forall q_{h} \in P_{h}
\end{aligned}
$$

where $\boldsymbol{R}^{n}=D_{\tau} \boldsymbol{u}^{n}-\boldsymbol{u}_{t}^{n}+\left(\left(\boldsymbol{u}^{n-1}-\boldsymbol{u}^{n}\right) \cdot \nabla\right) \boldsymbol{u}^{n}$. Taking $\boldsymbol{v}_{h}=D_{\tau} \boldsymbol{\theta}^{n}$ and $q_{h}=\eta^{n}$ in (3.2)-(3.3) yields

$$
\begin{aligned}
& \left\|D_{\tau} \boldsymbol{\theta}^{n}\right\|_{0}^{2}+\frac{\nu}{2 \tau}\left(\left\|\boldsymbol{\theta}^{n}\right\|_{h}^{2}-\left\|\boldsymbol{\theta}^{n-1}\right\|_{h}^{2}+\left\|\boldsymbol{\theta}^{n}-\boldsymbol{\theta}^{n-1}\right\|_{h}^{2}\right) \\
& =-\left(D_{\tau} \boldsymbol{\rho}^{n}, D_{\tau} \boldsymbol{\theta}^{n}\right)-\nu\left(\nabla \boldsymbol{\rho}^{n}, \nabla D_{\tau} \boldsymbol{\theta}^{n}\right)_{h}+\left(\xi^{n}, \nabla \cdot D_{\tau} \boldsymbol{\theta}^{n}\right)_{h} \\
& \quad-\left(\nabla \cdot D_{\tau} \boldsymbol{\rho}^{n}, \eta^{n}\right)_{h}+\left(\boldsymbol{R}^{n}, D_{\tau} \boldsymbol{\theta}^{n}\right)-\left(\left(\boldsymbol{u}^{n-1} \cdot \nabla\right) \boldsymbol{u}^{n}-\left(\boldsymbol{U}_{h}^{n-1} \cdot \nabla\right) \boldsymbol{U}_{h}^{n}, D_{\tau} \boldsymbol{\theta}^{n}\right)_{h} \\
& \quad+\sum_{e} \int_{\partial e}\left(\nu \frac{\partial \boldsymbol{u}^{n}}{\partial \boldsymbol{n}}-p^{n} \cdot \boldsymbol{n}\right) D_{\tau} \boldsymbol{\theta}^{n} d s:=\sum_{i=1}^{7} E_{i}\left(D_{\tau} \boldsymbol{\theta}^{n}\right) .
\end{aligned}
$$


Firstly, by Cauchy-Schwarz inequality and $\epsilon$-Young inequality, it is easy to check that

$$
E_{1}\left(D_{\tau} \boldsymbol{\theta}^{n}\right) \leq C h^{2} \tau^{-1} \int_{t_{n-1}}^{t_{n}}\left\|\boldsymbol{u}_{t}\right\|_{2} d t\left\|D_{\tau} \boldsymbol{\theta}^{n}\right\|_{0} \leq C h^{4} \tau^{-1} \int_{t_{n-1}}^{t_{n}}\left\|\boldsymbol{u}_{t}\right\|_{2}^{2} d t+\epsilon\left\|D_{\tau} \boldsymbol{\theta}^{n}\right\|_{0}^{2},
$$

and

$$
E_{5}\left(D_{\tau} \boldsymbol{\theta}^{n}\right) \leq C \tau \int_{t_{n-1}}^{t_{n}}\left(\left\|\boldsymbol{u}_{t}\right\|_{0}^{2}+\left\|\boldsymbol{u}_{t t}\right\|_{0}^{2}\right) d t+\epsilon\left\|D_{\tau} \boldsymbol{\theta}^{n}\right\|_{0}^{2} .
$$

Secondly, by use of Lemma 2.2, we have

$$
E_{4}\left(D_{\tau} \boldsymbol{\theta}^{n}\right) \leq C h^{2} \tau^{-1} \int_{t_{n-1}}^{t_{n}}\left\|\boldsymbol{u}_{t}\right\|_{3} d t\left\|\eta^{n}\right\|_{0}
$$

Thirdly, by Lemma 2.1 and summation by parts with respect to time $t$, it follows that

$$
\begin{aligned}
E_{2}\left(D_{\tau} \boldsymbol{\theta}^{n}\right) & =-\frac{\nu}{\tau}\left[\left(\nabla \boldsymbol{\rho}^{n}, \nabla \boldsymbol{\theta}^{n}\right)_{h}-\left(\nabla \boldsymbol{\rho}^{n-1}, \nabla \boldsymbol{\theta}^{n-1}\right)_{h}\right]+\frac{\nu}{\tau}\left(\nabla\left(\boldsymbol{\rho}^{n}-\boldsymbol{\rho}^{n-1}\right), \nabla \boldsymbol{\theta}^{n-1}\right)_{h} \\
& \leq-\frac{\nu}{\tau}\left[\left(\nabla \boldsymbol{\rho}^{n}, \nabla \boldsymbol{\theta}^{n}\right)_{h}-\left(\nabla \boldsymbol{\rho}^{n-1}, \nabla \boldsymbol{\theta}^{n-1}\right)_{h}\right]+C h^{2} \tau^{-1} \int_{t_{n-1}}^{t_{n}}\left\|\boldsymbol{u}_{t}\right\|_{3} d t\left\|\boldsymbol{\theta}^{n-1}\right\|_{h} .
\end{aligned}
$$

In the same way,

$$
\begin{aligned}
& E_{3}\left(D_{\tau} \boldsymbol{\theta}^{n}\right)= \frac{1}{\tau}\left[\left(\xi^{n}, \nabla \cdot \boldsymbol{\theta}^{n}\right)_{h}-\left(\xi^{n-1}, \nabla \cdot \boldsymbol{\theta}^{n-1}\right)_{h}\right]-\frac{1}{\tau}\left(\xi^{n}-\xi^{n-1}, \nabla \cdot \boldsymbol{\theta}^{n-1}\right)_{h} \\
& \leq \frac{1}{\tau}\left[\left(\xi^{n}, \nabla \cdot \boldsymbol{\theta}^{n}\right)_{h}-\left(\xi^{n-1}, \nabla \cdot \boldsymbol{\theta}^{n-1}\right)_{h}\right]+C h^{2} \tau^{-1} \int_{t_{n-1}}^{t_{n}}\left\|p_{t}\right\|_{2} d t\left\|\boldsymbol{\theta}^{n-1}\right\|_{h}, \quad \\
& E_{7}\left(D_{\tau} \boldsymbol{\theta}^{n}\right)=\frac{1}{\tau}\left[\sum_{e} \int_{\partial e}\left(\nu \frac{\partial \boldsymbol{u}^{n}}{\partial \boldsymbol{n}}-p^{n} \cdot \boldsymbol{n}\right) \boldsymbol{\theta}^{n} d s-\sum_{e} \int_{\partial e}\left(\nu \frac{\partial \boldsymbol{u}^{n-1}}{\partial \boldsymbol{n}}-p^{n-1} \cdot \boldsymbol{n}\right) \boldsymbol{\theta}^{n-1} d s\right] \\
&-\frac{1}{\tau} \sum_{e} \int_{\partial e} \int_{t_{n-1}}^{t_{n}}\left(\nu \frac{\partial \boldsymbol{u}_{t}}{\partial \boldsymbol{n}}-p_{t} \cdot \boldsymbol{n}\right) d t \boldsymbol{\theta}^{n-1} d s \\
& \leq \frac{1}{\tau}\left[\sum_{e} \int_{\partial e}\left(\nu \frac{\partial \boldsymbol{u}^{n}}{\partial \boldsymbol{n}}-p^{n} \cdot \boldsymbol{n}\right) \boldsymbol{\theta}^{n} d s-\sum_{e} \int_{\partial e}\left(\nu \frac{\partial \boldsymbol{u}^{n-1}}{\partial \boldsymbol{n}}-p^{n-1} \cdot \boldsymbol{n}\right) \boldsymbol{\theta}^{n-1} d s\right] \\
&+C h^{2} \tau^{-1} \int_{t_{n-1}}^{t_{n}}\left\|\boldsymbol{u}_{t}\right\|_{3}+\left\|p_{t}\right\|_{2} d t\left\|\boldsymbol{\theta}^{n-1}\right\|_{h} .
\end{aligned}
$$

Finally, note that (2.7) and

$$
\begin{aligned}
& \left(\boldsymbol{u}^{n-1} \cdot \nabla\right) \boldsymbol{u}^{n}-\left(\boldsymbol{U}_{h}^{n-1} \cdot \nabla\right) \boldsymbol{U}_{h}^{n} \\
= & \left(\left(\boldsymbol{u}^{n-1}-\Pi_{h} \boldsymbol{u}^{n-1}\right) \cdot \nabla\right) \boldsymbol{u}^{n}+\left(\left(\Pi_{h} \boldsymbol{u}^{n-1}-\boldsymbol{U}_{h}^{n-1}\right) \cdot \nabla\right) \boldsymbol{u}^{n} \\
& +\left(\left(\boldsymbol{U}_{h}^{n-1}-\Pi_{h} \boldsymbol{u}^{n-1}\right) \cdot \nabla\right)\left(\boldsymbol{u}^{n}-\Pi_{h} \boldsymbol{u}^{n}\right)+\left(\left(\Pi_{h} \boldsymbol{u}^{n-1}-\boldsymbol{u}^{n-1}\right) \cdot \nabla\right)\left(\boldsymbol{u}^{n}-\Pi_{h} \boldsymbol{u}^{n}\right) \\
& \quad+\left(\boldsymbol{u}^{n-1} \cdot \nabla\right)\left(\boldsymbol{u}^{n}-\Pi_{h} \boldsymbol{u}^{n}\right)+\left(\boldsymbol{U}_{h}^{n-1} \cdot \nabla\right)\left(\Pi_{h} \boldsymbol{u}^{n}-\boldsymbol{U}_{h}^{n}\right):=\sum_{i=1}^{6} F_{i},
\end{aligned}
$$

we have

$$
\begin{aligned}
\left(F_{1}, D_{\tau} \boldsymbol{\theta}^{n}\right)_{h} & \leq\left\|\boldsymbol{u}^{n-1}-\Pi_{h} \boldsymbol{u}^{n-1}\right\|_{0}\left\|\nabla \boldsymbol{u}^{n}\right\|_{0, \infty}\left\|D_{\tau} \boldsymbol{\theta}^{n}\right\|_{0} \\
& \leq C h^{2}\left\|\boldsymbol{u}^{n-1}\right\|_{2}\left\|D_{\tau} \boldsymbol{\theta}^{n}\right\|_{0} \leq C h^{4}+\epsilon\left\|D_{\tau} \boldsymbol{\theta}^{n}\right\|_{0}^{2}, \\
\left(F_{2}, D_{\tau} \boldsymbol{\theta}^{n}\right)_{h} & \leq\left\|\Pi_{h} \boldsymbol{u}^{n-1}-\boldsymbol{U}_{h}^{n-1}\right\|_{0}\left\|\nabla \boldsymbol{u}^{n}\right\|_{0, \infty}\left\|D_{\tau} \boldsymbol{\theta}^{n}\right\|_{0} \\
& \leq C\left\|\boldsymbol{\theta}^{n}\right\|_{h}\left\|D_{\tau} \boldsymbol{\theta}^{n}\right\|_{0} \leq C\left\|\boldsymbol{\theta}^{n}\right\|_{h}^{2}+\epsilon\left\|D_{\tau} \boldsymbol{\theta}^{n}\right\|_{0}^{2},
\end{aligned}
$$




$$
\begin{aligned}
\left(F_{3}, D_{\tau} \boldsymbol{\theta}^{n}\right)_{h} & \leq\left\|\boldsymbol{\theta}^{n-1}\right\|_{0}\left\|\nabla\left(\boldsymbol{u}^{n}-\Pi_{h} \boldsymbol{u}^{n}\right)\right\|_{0}\left\|D_{\tau} \boldsymbol{\theta}^{n}\right\|_{0, \infty} \\
& \leq C\left\|\boldsymbol{\theta}^{n-1}\right\|_{h}\left(h\left\|\boldsymbol{u}^{n}\right\|_{2}\right)\left(h^{-1}\left\|D_{\tau} \boldsymbol{\theta}^{n}\right\|_{0}\right) \\
& \leq C\left\|\boldsymbol{\theta}^{n-1}\right\|_{h}\left\|D_{\tau} \boldsymbol{\theta}^{n}\right\|_{0} \leq C\left\|\boldsymbol{\theta}^{n-1}\right\|_{h}^{2}+\epsilon\left\|D_{\tau} \boldsymbol{\theta}^{n}\right\|_{0}^{2}, \\
\left(F_{4}, D_{\tau} \boldsymbol{\theta}^{n}\right)_{h} & \leq\left\|\Pi_{h} \boldsymbol{u}^{n-1}-\boldsymbol{u}^{n-1}\right\|_{0}\left\|\nabla\left(\boldsymbol{u}^{n}-\Pi_{h} \boldsymbol{u}^{n}\right)\right\|_{0}\left\|D_{\tau} \boldsymbol{\theta}^{n}\right\|_{0, \infty} \\
& \leq C h^{2}\left\|\boldsymbol{u}^{n-1}\right\|_{2}\left(h\left\|\boldsymbol{u}^{n}\right\|_{2}\right)\left(h^{-1}\left\|D_{\tau} \boldsymbol{\theta}^{n}\right\|_{0}\right) \\
& \leq C h^{2}\left\|\boldsymbol{u}^{n-1}\right\|_{2}\left\|D_{\tau} \boldsymbol{\theta}^{n}\right\|_{0} \leq C h^{4}+\epsilon\left\|D_{\tau} \boldsymbol{\theta}^{n}\right\|_{0}^{2} .
\end{aligned}
$$

As for $\left(F_{5}, D_{\tau} \boldsymbol{\theta}^{n}\right)_{h}$, we rewrite it as

$$
\begin{aligned}
\left(F_{5}, D_{\tau} \boldsymbol{\theta}^{n}\right)_{h}=\frac{1}{\tau}[ & \left.\left(\left(\boldsymbol{u}^{n-1} \cdot \nabla\right)\left(\boldsymbol{u}^{n}-\Pi_{h} \boldsymbol{u}^{n}\right), \boldsymbol{\theta}^{n}\right)_{h}-\left(\left(\boldsymbol{u}^{n-2} \cdot \nabla\right)\left(\boldsymbol{u}^{n-1}-\Pi_{h} \boldsymbol{u}^{n-1}\right), \boldsymbol{\theta}^{n-1}\right)_{h}\right] \\
& -\frac{1}{\tau}\left(\left(\left(\boldsymbol{u}^{n-1} \cdot \nabla\right)\left(\boldsymbol{u}^{n}-\Pi_{h} \boldsymbol{u}^{n}\right)\right)-\left(\left(\boldsymbol{u}^{n-2} \cdot \nabla\right)\left(\boldsymbol{u}^{n-1}-\Pi_{h} \boldsymbol{u}^{n-1}\right)\right), \boldsymbol{\theta}^{n-1}\right)_{h} \\
=\frac{1}{\tau} & {\left[\left(\left(\boldsymbol{u}^{n-1} \cdot \nabla\right)\left(\boldsymbol{u}^{n}-\Pi_{h} \boldsymbol{u}^{n}\right), \boldsymbol{\theta}^{n}\right)_{h}-\left(\left(\boldsymbol{u}^{n-2} \cdot \nabla\right)\left(\boldsymbol{u}^{n-1}-\Pi_{h} \boldsymbol{u}^{n-1}\right), \boldsymbol{\theta}^{n-1}\right)_{h}\right] } \\
& -\left(\left(\frac{\boldsymbol{u}^{n-1}-\boldsymbol{u}^{n-2}}{\tau} \cdot \nabla\right)\left(\boldsymbol{u}^{n}-\Pi_{h} \boldsymbol{u}^{n}\right), \boldsymbol{\theta}^{n-1}\right)_{h} \\
& -\left(\boldsymbol{u}^{n-2} \cdot \nabla\right)\left(\nabla\left(\frac{\boldsymbol{u}^{n}-\boldsymbol{u}^{n-1}}{\tau}-\Pi_{h} \frac{\boldsymbol{u}^{n}-\boldsymbol{u}^{n-1}}{\tau}\right), \boldsymbol{\theta}^{n-1}\right)_{h} .
\end{aligned}
$$

Moreover, we introduce the local $L^{2}$ projection defined by

$$
\mathcal{P}_{e} \boldsymbol{u}=\left.\overline{\boldsymbol{u}}\right|_{e}=\left.\boldsymbol{u}\right|_{e}=\frac{1}{|e|} \int_{e} \boldsymbol{u} d \boldsymbol{x}, \quad \forall e \in \mathcal{T}_{h} .
$$

Then it follows that

$$
\left\|\mathcal{P}_{e} \boldsymbol{u}\right\|_{0, e} \leq\|\boldsymbol{u}\|_{0, e}, \quad\left\|\boldsymbol{u}-\mathcal{P}_{e} \boldsymbol{u}\right\|_{0, e} \leq C h\|\boldsymbol{u}\|_{1, e}, \quad \text { for } \boldsymbol{u} \in\left(H^{1}(e)\right)^{2} .
$$

Denote $\boldsymbol{Z}=\left(\boldsymbol{u}^{n-1}-\boldsymbol{u}^{n-2}\right) / \tau$. Thus we have

$$
\begin{aligned}
& \left((\boldsymbol{Z} \cdot \nabla)\left(\boldsymbol{u}^{n}-\Pi_{h} \boldsymbol{u}^{n}\right), \boldsymbol{\theta}^{n-1}\right)_{h} \\
= & \sum_{e \in \mathcal{T}_{h}}\left((\boldsymbol{Z} \cdot \nabla)\left(\boldsymbol{u}^{n}-\Pi_{h} \boldsymbol{u}^{n}\right), \boldsymbol{\theta}^{n-1}\right)_{e}=\sum_{e \in \mathcal{T}_{h}}\left((\boldsymbol{Z} \cdot \nabla)\left(\boldsymbol{u}^{n}-\Pi_{h} \boldsymbol{u}^{n}\right), \boldsymbol{\theta}^{n-1}-\mathcal{P}_{e} \boldsymbol{\theta}^{n-1}\right)_{e} \\
& +\sum_{e \in \mathcal{T}_{h}}\left(((\boldsymbol{Z}-\overline{\boldsymbol{Z}}) \cdot \nabla)\left(\boldsymbol{u}^{n}-\Pi_{h} \boldsymbol{u}^{n}\right), \mathcal{P}_{e} \boldsymbol{\theta}^{n-1}\right)_{e}+\sum_{e \in \mathcal{T}_{h}}\left((\overline{\boldsymbol{Z}} \cdot \nabla)\left(\boldsymbol{u}^{n}-\Pi_{h} \boldsymbol{u}^{n}\right), \mathcal{P}_{e} \boldsymbol{\theta}^{n-1}\right)_{e}:=\sum_{i=1}^{3} A_{i} .
\end{aligned}
$$

By Lemma 2.1, (2.7) and (3.17), we have

$$
\begin{aligned}
A_{1} & \leq \sum_{e \in \mathcal{T}_{h}}\|\boldsymbol{Z}\|_{0, \infty, e}\left\|\nabla\left(\boldsymbol{u}^{n}-\Pi_{h} \boldsymbol{u}^{n}\right)\right\|_{0, e}\left\|\boldsymbol{\theta}^{n-1}-\mathcal{P}_{e} \boldsymbol{\theta}^{n-1}\right\|_{0, e} \\
& \leq \sum_{e \in \mathcal{T}_{h}} C_{e} h^{2}\left\|\boldsymbol{u}^{n}\right\|_{2, e}\left\|\boldsymbol{\theta}^{n-1}\right\|_{1, e} \leq C h^{2}\left\|\boldsymbol{\theta}^{n-1}\right\|_{h}, \\
A_{2} & \leq \sum_{e \in \mathcal{T}_{h}}\|\boldsymbol{Z}-\overline{\boldsymbol{Z}}\|_{0, \infty, e}\left\|\nabla\left(\boldsymbol{u}^{n}-\Pi_{h} \boldsymbol{u}^{n}\right)\right\|_{0, e}\left\|\mathcal{P}_{e} \boldsymbol{\theta}^{n-1}\right\|_{0, e} \\
& \leq \sum_{e \in \mathcal{T}_{h}} C_{e} h^{2}\|\boldsymbol{Z}\|_{1, \infty, e}\left\|\boldsymbol{u}^{n}\right\|_{2, e}\left\|\boldsymbol{\theta}^{n-1}\right\|_{0, e} \leq C h^{2}\left\|\boldsymbol{\theta}^{n-1}\right\|_{h},
\end{aligned}
$$


and

$$
A_{3} \leq \sum_{e \in \mathcal{T}_{h}} C_{e} h^{2}\left\|\boldsymbol{u}^{n}\right\|_{3, e}\left\|\mathcal{P}_{e} \boldsymbol{\theta}^{n-1}\right\|_{0, e} \leq C h^{2}\left\|\boldsymbol{\theta}^{n-1}\right\|_{h}
$$

Therefore, there holds

$$
\left((\boldsymbol{Z} \cdot \nabla)\left(\boldsymbol{u}^{n}-\Pi_{h} \boldsymbol{u}^{n}\right), \boldsymbol{\theta}^{n-1}\right)_{h} \leq C h^{2}\left\|\boldsymbol{\theta}^{n-1}\right\|_{h} \leq C h^{4}+C\left\|\boldsymbol{\theta}^{n-1}\right\|_{h}^{2} .
$$

In the same way,

$$
\left(\boldsymbol{u}^{n-2} \cdot \nabla\right)\left(\nabla\left(\frac{\boldsymbol{u}^{n}-\boldsymbol{u}^{n-1}}{\tau}-\Pi_{h} \frac{\boldsymbol{u}^{n}-\boldsymbol{u}^{n-1}}{\tau}\right), \boldsymbol{\theta}^{n-1}\right)_{h} \leq C h^{4}+C\left\|\boldsymbol{\theta}^{n-1}\right\|_{h}^{2} .
$$

Thus, we have

$$
\begin{aligned}
\left(F_{5}, D_{\tau} \boldsymbol{\theta}^{n}\right)_{h} \leq \frac{1}{\tau} & {\left[\left(\left(\boldsymbol{u}^{n-1} \cdot \nabla\right)\left(\boldsymbol{u}^{n}-\Pi_{h} \boldsymbol{u}^{n}\right), \boldsymbol{\theta}^{n}\right)_{h}-\left(\left(\boldsymbol{u}^{n-2} \cdot \nabla\right)\left(\boldsymbol{u}^{n-1}-\Pi_{h} \boldsymbol{u}^{n-1}\right), \boldsymbol{\theta}^{n-1}\right)_{h}\right] } \\
& +C h^{4}+C\left\|\boldsymbol{\theta}^{n-1}\right\|_{h}^{2}
\end{aligned}
$$

As for $\left(F_{6}, D_{\tau} \boldsymbol{\theta}^{n}\right)_{h}$, we need the following induction hypothesis and prove it later,

$$
\left\|\boldsymbol{U}_{h}^{n}\right\|_{0, \infty} \leq K, \quad n=0,1, \ldots, N
$$

where $K=\left\|\Pi_{h} \boldsymbol{u}\right\|_{L^{\infty}\left(L^{\infty}\right)}+1$.

In fact, for $n=0, \boldsymbol{U}_{h}^{0}=\Pi_{h} \boldsymbol{u}_{0}$, we have $\left\|\boldsymbol{U}_{h}^{0}\right\|_{0, \infty} \leq K$. We assume that (3.25) holds for $n=0,1, \ldots, k-1$, for $k>0$, then we have

$$
\left(F_{6}, D_{\tau} \boldsymbol{\theta}^{n}\right)_{h} \leq\left\|\boldsymbol{U}_{h}^{n-1}\right\|_{0, \infty}\left\|\boldsymbol{\theta}^{n}\right\|_{h}\left\|D_{\tau} \boldsymbol{\theta}^{n}\right\|_{0} \leq C\left\|\boldsymbol{\theta}^{n}\right\|_{h}^{2}+\epsilon\left\|D_{\tau} \boldsymbol{\theta}^{n}\right\|_{0}^{2} .
$$

With the estimates of $F_{1}-F_{6}$, it follows that

$$
\begin{aligned}
E_{6}\left(D_{\tau} \boldsymbol{\theta}^{n}\right) \leq \frac{1}{\tau} & {\left[\left(\left(\boldsymbol{u}^{n-1} \cdot \nabla\right)\left(\boldsymbol{u}^{n}-\Pi_{h} \boldsymbol{u}^{n}\right), \boldsymbol{\theta}^{n}\right)_{h}-\left(\left(\boldsymbol{u}^{n-2} \cdot \nabla\right)\left(\boldsymbol{u}^{n-1}-\Pi_{h} \boldsymbol{u}^{n-1}\right), \boldsymbol{\theta}^{n-1}\right)_{h}\right] } \\
& +C h^{4}+C\left(\left\|\boldsymbol{\theta}^{n}\right\|_{h}^{2}+\left\|\boldsymbol{\theta}^{n-1}\right\|_{h}^{2}\right)+5 \epsilon\left\|D_{\tau} \boldsymbol{\theta}^{n}\right\|_{0}^{2}
\end{aligned}
$$

Substituting (3.5)-(3.10) and (3.27) into (3.4) leads to

$$
\begin{aligned}
& \left\|D_{\tau} \boldsymbol{\theta}^{n}\right\|_{0}^{2}+\frac{\nu}{2 \tau}\left(\left\|\boldsymbol{\theta}^{n}\right\|_{h}^{2}-\left\|\boldsymbol{\theta}^{n-1}\right\|_{h}^{2}\right) \\
& \leq C h^{4}+C h^{4} \tau^{-1} \int_{t_{n-1}}^{t_{n}}\left\|\boldsymbol{u}_{t}\right\|_{3}^{2}+\left\|p_{t}\right\|_{2}^{2} d t+C \tau \int_{t_{n-1}}^{t_{n}}\left\|\boldsymbol{u}_{t}\right\|_{0}^{2}+\left\|\boldsymbol{u}_{t t}\right\|_{0}^{2} d t \\
& +C h^{2} \tau^{-1} \int_{t_{n-1}}^{t_{n}}\left\|\boldsymbol{u}_{t}\right\|_{3} d t\left\|\eta^{n}\right\|_{0}+C\left(\left\|\boldsymbol{\theta}^{n}\right\|_{h}^{2}+\left\|\boldsymbol{\theta}^{n-1}\right\|_{h}^{2}\right)+7 \epsilon\left\|D_{\tau} \boldsymbol{\theta}^{n}\right\|_{0}^{2} \\
& \quad-\frac{\nu}{\tau}\left[\left(\nabla \boldsymbol{\rho}^{n}, \nabla \boldsymbol{\theta}^{n}\right)_{h}-\left(\nabla \boldsymbol{\rho}^{n-1}, \nabla \boldsymbol{\theta}^{n-1}\right)_{h}\right]+\frac{1}{\tau}\left[\left(\xi^{n}, \nabla \cdot \boldsymbol{\theta}^{n}\right)_{h}-\left(\xi^{n-1}, \nabla \cdot \boldsymbol{\theta}^{n-1}\right)_{h}\right] \\
& +\frac{1}{\tau}\left[\left(\left(\boldsymbol{u}^{n-1} \cdot \nabla\right)\left(\boldsymbol{u}^{n}-\Pi_{h} \boldsymbol{u}^{n}\right), \boldsymbol{\theta}^{n}\right)_{h}-\left(\left(\boldsymbol{u}^{n-2} \cdot \nabla\right)\left(\boldsymbol{u}^{n-1}-\Pi_{h} \boldsymbol{u}^{n-1}\right), \boldsymbol{\theta}^{n-1}\right)_{h}\right] \\
& +\frac{1}{\tau}\left[\sum_{e} \int_{\partial e}\left(\nu \frac{\partial \boldsymbol{u}^{n}}{\partial \boldsymbol{n}}-p^{n} \cdot \boldsymbol{n}\right) \boldsymbol{\theta}^{n} d s-\sum_{e} \int_{\partial e}\left(\nu \frac{\partial \boldsymbol{u}^{n-1}}{\partial \boldsymbol{n}}-p^{n-1} \cdot \boldsymbol{n}\right) \boldsymbol{\theta}^{n-1} d s\right] .
\end{aligned}
$$


On the other hand, we rewrite (3.2) as

$$
\begin{aligned}
\left(\eta^{n}, \nabla \cdot \boldsymbol{v}_{h}\right)_{h}= & \left(D_{\tau} \boldsymbol{\theta}^{n}, \boldsymbol{v}_{h}\right)+\nu\left(\nabla \boldsymbol{\theta}^{n}, \nabla \boldsymbol{v}_{h}\right)_{h}+\left(D_{\tau} \boldsymbol{\rho}^{n}, \boldsymbol{v}_{h}\right)+\nu\left(\nabla \boldsymbol{\rho}^{n}, \nabla \boldsymbol{v}_{h}\right)_{h} \\
& -\left(\xi^{n}, \nabla \cdot \boldsymbol{v}_{h}\right)_{h}-\left(\boldsymbol{R}^{n}, \boldsymbol{v}_{h}\right)-\left(\left(\boldsymbol{u}^{n-1} \cdot \nabla\right) \boldsymbol{u}^{n}-\left(\boldsymbol{U}_{h}^{n-1} \cdot \nabla\right) \boldsymbol{U}_{h}^{n}, \boldsymbol{v}_{h}\right)_{h} \\
& -\sum_{e} \int_{\partial e}\left(\nu \frac{\partial \boldsymbol{u}^{n}}{\partial \boldsymbol{n}}-p^{n} \cdot \boldsymbol{n}\right) \boldsymbol{v}_{h} d s
\end{aligned}
$$

Thus, we have

$$
\begin{aligned}
& \left(D_{\tau} \boldsymbol{\theta}^{n}, \boldsymbol{v}_{h}\right) \leq C\left\|D_{\tau} \boldsymbol{\theta}^{n}\right\|_{0}\left\|\boldsymbol{v}_{h}\right\|_{h}, \quad\left(D_{\tau} \boldsymbol{\rho}^{n}, \boldsymbol{v}_{h}\right) \leq C h^{2}\left\|\boldsymbol{u}_{t}\right\|_{L^{\infty}\left(H^{2}\right)}\left\|\boldsymbol{v}_{h}\right\|_{h}, \\
& \left(\nabla \boldsymbol{\theta}^{n}, \nabla \boldsymbol{v}_{h}\right)_{h} \leq\left\|\boldsymbol{\theta}^{n}\right\|_{h}\|\boldsymbol{v}\|_{h}, \quad\left(\nabla \boldsymbol{\rho}^{n}, \nabla \boldsymbol{v}_{h}\right)_{h} \leq C h^{2}\left\|\boldsymbol{u}^{n}\right\|_{3}\left\|\boldsymbol{v}_{h}\right\|_{h}, \\
& \left(\boldsymbol{R}^{n}, \boldsymbol{v}_{h}\right) \leq C \tau\left(\left\|\boldsymbol{u}_{t}\right\|_{L^{\infty}\left(L^{2}\right)}+\left\|\boldsymbol{u}_{t t}\right\|_{L^{\infty}\left(L^{2}\right)}\right)\left\|\boldsymbol{v}_{h}\right\|_{h}, \quad\left(\xi^{n}, \nabla \cdot \boldsymbol{v}_{h}\right)_{h} \leq C h^{2}\left\|p^{n}\right\|_{2}\left\|\boldsymbol{v}_{h}\right\|_{h} \\
& \sum_{e} \int_{\partial e}\left(\nu \frac{\partial \boldsymbol{u}^{n}}{\partial \boldsymbol{n}}-p^{n} \cdot \boldsymbol{n}\right) \boldsymbol{v}_{h} d s \leq C h^{2}\left(\left\|\boldsymbol{u}^{n}\right\|_{3}+\left\|p^{n}\right\|_{2}\right)\left\|\boldsymbol{v}_{h}\right\|_{h} .
\end{aligned}
$$

In addition, according to (3.11), it follows that

$$
\left(\left(\boldsymbol{u}^{n-1} \cdot \nabla\right) \boldsymbol{u}^{n}-\left(\boldsymbol{U}_{h}^{n-1} \cdot \nabla\right) \boldsymbol{U}_{h}^{n}, \boldsymbol{v}_{h}\right)_{h} \leq C\left(h^{2}+\left\|\boldsymbol{\theta}^{n}\right\|_{h}+\left\|\boldsymbol{\theta}^{n-1}\right\|_{h}\right)\left\|\boldsymbol{v}_{h}\right\|_{h}
$$

Therefore, by the discrete LBB condition, we have

$$
\begin{aligned}
\beta\left\|J_{h} p^{n}-P_{h}^{n}\right\|_{0} & \leq \sup _{\boldsymbol{v}_{h} \in \boldsymbol{V}_{h}} \frac{\left(\nabla \cdot \boldsymbol{v}_{h}, J_{h} p^{n}-P_{h}^{n}\right)}{\left\|\boldsymbol{v}_{h}\right\|_{h}} \\
& \leq C\left(h^{2}+\tau\right)+C\left\|D_{\tau} \boldsymbol{\theta}^{n}\right\|_{0}+C\left(\left\|\boldsymbol{\theta}^{n}\right\|_{h}+\left\|\boldsymbol{\theta}^{n-1}\right\|_{h}\right) .
\end{aligned}
$$

Now, substituting (3.30) into (3.28) and using $\epsilon$-Young inequality, we have

$$
\begin{aligned}
\left\|D_{\tau} \boldsymbol{\theta}^{n}\right\|_{0}^{2}+\frac{\nu}{2 \tau}\left(\left\|\boldsymbol{\theta}^{n}\right\|_{h}^{2}-\left\|\boldsymbol{\theta}^{n-1}\right\|_{h}^{2}\right) \\
\leq C\left(h^{4}+\tau^{2}\right)+C h^{4} \tau^{-1} \int_{t_{n-1}}^{t_{n}}\left\|\boldsymbol{u}_{t}\right\|_{3}^{2}+\left\|p_{t}\right\|_{2}^{2} d t+C\left(\left\|\boldsymbol{\theta}^{n}\right\|_{h}^{2}+\left\|\boldsymbol{\theta}^{n-1}\right\|_{h}^{2}\right)+9 \epsilon\left\|D_{\tau} \boldsymbol{\theta}^{n}\right\|_{0}^{2} \\
\quad-\frac{\nu}{\tau}\left[\left(\nabla \boldsymbol{\rho}^{n}, \nabla \boldsymbol{\theta}^{n}\right)_{h}-\left(\nabla \boldsymbol{\rho}^{n-1}, \nabla \boldsymbol{\theta}^{n-1}\right)_{h}\right]+\frac{1}{\tau}\left[\left(\xi^{n}, \nabla \cdot \boldsymbol{\theta}^{n}\right)_{h}-\left(\xi^{n-1}, \nabla \cdot \boldsymbol{\theta}^{n-1}\right)_{h}\right] \\
+\frac{1}{\tau}\left[\left(\left(\boldsymbol{u}^{n-1} \cdot \nabla\right)\left(\boldsymbol{u}^{n}-\Pi_{h} \boldsymbol{u}^{n}\right), \boldsymbol{\theta}^{n}\right)_{h}-\left(\left(\boldsymbol{u}^{n-2} \cdot \nabla\right)\left(\boldsymbol{u}^{n-1}-\Pi_{h} \boldsymbol{u}^{n-1}\right), \boldsymbol{\theta}^{n-1}\right)_{h}\right] \\
+\frac{1}{\tau}\left[\sum_{e} \int_{\partial e}\left(\nu \frac{\partial \boldsymbol{u}^{n}}{\partial \boldsymbol{n}}-p^{n} \cdot \boldsymbol{n}\right) \boldsymbol{\theta}^{n} d s-\sum_{e} \int_{\partial e}\left(\nu \frac{\partial \boldsymbol{u}^{n-1}}{\partial \boldsymbol{n}}-p^{n-1} \cdot \boldsymbol{n}\right) \boldsymbol{\theta}^{n-1} d s\right] .
\end{aligned}
$$

Then, summing up the above inequality and noting that $\boldsymbol{\theta}^{n}=0$ shows that

$$
\begin{aligned}
& \frac{\tau}{2} \sum_{i=1}^{n}\left\|D_{\tau} \boldsymbol{\theta}^{i}\right\|_{0}^{2}+\frac{\nu}{2}\left\|\boldsymbol{\theta}^{n}\right\|_{h}^{2} \\
& \leq C\left(h^{4}+\tau^{2}\right)+C \tau \sum_{i=1}^{n}\left\|\boldsymbol{\theta}^{i}\right\|_{h}^{2}-\nu\left(\nabla \boldsymbol{\rho}^{n}, \nabla \boldsymbol{\theta}^{n}\right)_{h}+\left(\left(\boldsymbol{u}^{n-1} \cdot \nabla\right)\left(\boldsymbol{u}^{n}-\Pi_{h} \boldsymbol{u}^{n}\right), \boldsymbol{\theta}^{n}\right)_{h} \\
& \quad+\left(\xi^{n}, \nabla \cdot \boldsymbol{\theta}^{n}\right)_{h}+\sum_{e} \int_{\partial e}\left(\nu \frac{\partial \boldsymbol{u}^{n}}{\partial \boldsymbol{n}}-p^{n} \cdot \boldsymbol{n}\right) \boldsymbol{\theta}^{n} d s .
\end{aligned}
$$


Furthermore, using Lemmas 2.1-2.2 and the estimation process as $F_{5}$ again, we have

$$
\begin{aligned}
& \frac{\tau}{2} \sum_{i=1}^{n}\left\|D_{\tau} \boldsymbol{\theta}^{i}\right\|_{0}^{2}+\frac{\nu}{2}\left\|\boldsymbol{\theta}^{n}\right\|_{h}^{2} \\
\leq & C\left(h^{4}+\tau^{2}\right)+C \tau \sum_{i=1}^{n}\left\|\boldsymbol{\theta}^{i}\right\|_{h}^{2}+C h^{4}\left(\left\|\boldsymbol{u}^{n}\right\|_{3}^{2}+\|p\|_{2}^{2}\right)+\frac{\nu}{4}\left\|\boldsymbol{\theta}^{n}\right\|_{h}^{2},
\end{aligned}
$$

which implies that

$$
\left\|\boldsymbol{\theta}^{n}\right\|_{h}^{2}+\tau \sum_{i=1}^{n}\left\|D_{\tau} \boldsymbol{\theta}^{n}\right\|_{0}^{2} \leq C\left(h^{4}+\tau^{2}\right)+C \tau \sum_{i=1}^{n}\left\|\boldsymbol{\theta}^{i}\right\|_{h}^{2} .
$$

Thanks to Lemma 2.3, there exists a small $\tau_{1}$, when $\tau<\tau_{1}$, we have

$$
\left\|\boldsymbol{\theta}^{n}\right\|_{h}^{2}+\tau \sum_{i=1}^{n}\left\|D_{\tau} \boldsymbol{\theta}^{n}\right\|_{0}^{2} \leq C\left(h^{4}+\tau^{2}\right)
$$

Now, we are in the position to prove (3.25). In fact, by (3.35) and inverse inequality, we have for $n=k$

$$
\begin{aligned}
\left\|\boldsymbol{U}_{h}^{n}\right\|_{0, \infty} & \leq\left\|\Pi_{h} \boldsymbol{u}^{n}\right\|_{0, \infty}+\left\|\Pi_{h} \boldsymbol{u}^{n}-\boldsymbol{U}_{h}^{n}\right\|_{0, \infty} \leq\left\|\Pi_{h} \boldsymbol{u}\right\|_{L^{\infty}\left(L^{\infty}\right)}+C h^{-1}\left\|\Pi_{h} \boldsymbol{u}^{n}-\boldsymbol{U}_{h}^{n}\right\|_{0} \\
& \leq\left\|\Pi_{h} \boldsymbol{u}\right\|_{L^{\infty}\left(L^{\infty}\right)}+C h^{-1}\left\|\Pi_{h} \boldsymbol{u}^{n}-\boldsymbol{U}_{h}^{n}\right\|_{h} \leq\left\|\Pi_{h} \boldsymbol{u}\right\|_{L^{\infty}\left(L^{\infty}\right)}+C h^{-1}\left(h^{2}+\tau\right) \\
& \leq\left\|\Pi_{h} \boldsymbol{u}\right\|_{L^{\infty}\left(L^{\infty}\right)}+1,
\end{aligned}
$$

where we require $\tau=O\left(h^{1+\gamma}\right), \gamma>0$ and $C\left(h+h^{\gamma}\right) \leq 1$. Then, the induction hypothesis (3.25) holds true uniformly for $n=0,1, \cdots, N$.

Finally, substituting (3.35) into (3.30), it follows that

$$
\left\|J_{h} p^{n}-P_{h}^{n}\right\|_{0} \leq C\left(h^{2}+\tau\right)+C\left\|D_{\tau} \boldsymbol{\theta}^{n}\right\|_{0} .
$$

Using (3.35) again leads to

$$
\tau \sum_{i=1}^{n}\left\|J_{h} p^{i}-P_{h}^{i}\right\|_{0}^{2} \leq C\left(h^{4}+\tau^{2}\right)+C \tau \sum_{i=1}^{n}\left\|D_{\tau} \boldsymbol{\theta}^{i}\right\|_{0}^{2} \leq C\left(h^{4}+\tau^{2}\right),
$$

which together with (3.35) completes the proof.

Remark 3.1. We can see that the superclose error estimate $\left\|\Pi_{h} \boldsymbol{u}^{n}-\boldsymbol{U}_{h}^{n}\right\|_{h} \leq C\left(h^{2}+\tau\right)$ in the above proof indeed plays a key role to bound the numerical solution $\boldsymbol{U}_{h}^{n}$ in $L^{\infty}$-norm.

In what follows, we introduce the postprocessing operator $\Pi_{2 h}$ to get the global superconvergent estimates (see $[32,33]$ for details). Let $\mathcal{T}_{h}$ be obtained from a coarse mesh $\mathcal{T}_{2 h}$ by bi-sectioning each rectangle $\tilde{e}$ and $a_{i}, i=1,2, \cdots, 9$ be the nodes on $\tilde{e}$ (see Fig. 2.1).

For any $v_{h} \in C R_{0}^{h}$, it has the form

$$
\left.v_{h}\right|_{\tilde{e}}=\sum_{i=1}^{9} v_{i} \phi_{i},
$$


where $\phi_{i}, i=1,2, \cdots, 9$ are the basis functions in $C R_{0}^{h}$. Now, we define the interpolation operator $\Pi_{2 h} v_{h} \in Q_{2}(\tilde{e})$ by

$$
\Pi_{2 h}=\sum_{i=1}^{9} v_{i} \Phi_{i}
$$

where $\Phi_{i}, 1 \leq i \leq 9$, are the basis functions of the space $Q_{2}(\tilde{e})$. Moreover, for $w \in H^{2}(\Omega) \cap$ $H_{0}^{1}(\Omega)$, let $\Pi_{2 h}^{*} w$ be its piecewise Lagrange biquadratic interpolation with respect to the coarse mesh $\mathcal{T}_{2 h}$ defined by

$$
\left.\Pi_{2 h}^{*}\right|_{\tilde{e}}=\sum_{i=1}^{9} w_{i} \Phi_{i}
$$

where $w_{i}$ are the values of $w$ on the nodes $a_{i}, i=1,2, \cdots, 9$.

Then [32] and [33] have shown that

$$
\begin{array}{ll}
\Pi_{2 h} \Pi_{h} u=\Pi_{2 h}^{*} u, & \forall u \in H^{2}(\Omega) \cap H_{0}^{1}(\Omega), \\
\left|\Pi_{2 h} v_{h}\right|_{1} \leq C\left\|v_{h}\right\|_{h}, & \forall v_{h} \in C R_{0}^{h}, \\
\left|\Pi_{2 h}^{*} u-u\right|_{1} \leq C h^{2}\|u\|_{3}, & \forall u \in H^{3}(\Omega) .
\end{array}
$$

Moreover, let $J_{2 h}: p \in H^{1}(\Omega) \rightarrow J_{2 h} p \in \mathcal{Q}_{1}(\tilde{e})$ satisfy $\int_{e_{j}}\left(p-J_{2 h} p\right) d x_{1} d x_{2}=0$, where $e_{j}(j=1,2,3,4)$ are the four small elements of the macroelement $\tilde{e}$ (see Fig. 2.1). Then the following properties hold

$$
\begin{array}{ll}
J_{2 h} J_{h} p=J_{2 h} p, & \forall p \in H^{1}(\Omega), \\
\left\|J_{2 h} p_{h}\right\|_{0} \leq C\left\|p_{h}\right\|_{0}, & \forall p_{h} \in P_{h}, \\
\left\|p-J_{2 h} p\right\|_{0} \leq C h^{2}\|p\|_{2}, & \forall p \in H^{2}(\Omega) .
\end{array}
$$

Based on the above interpolation postprocessing operators $\Pi_{2 h}$ and $J_{2 h}$, we can get the following superconvergence results.

Theorem 3.2. Under the conditions of Theorem 3.1 , for $n=1, \ldots, N$, we have

$$
\left|\boldsymbol{u}^{n}-\Pi_{2 h} \boldsymbol{U}_{h}^{n}\right|_{1} \leq C\left(h^{2}+\tau\right), \quad \tau \sum_{i=1}^{n}\left\|p^{i}-J_{2 h} P_{h}^{i}\right\|_{0}^{2} \leq C\left(h^{4}+\tau^{2}\right) .
$$

Proof. It follows from properties (3.39)-(3.41) and Theorem 3.1 that

$$
\begin{aligned}
& \left|\boldsymbol{u}^{n}-\Pi_{2 h} \boldsymbol{U}_{h}^{n}\right|_{1} \\
\leq & \left|\boldsymbol{u}^{n}-\Pi_{2 h}^{*} \boldsymbol{u}^{n}\right|_{1}+\left|\Pi_{2 h}^{*} \boldsymbol{u}^{n}-\Pi_{2 h} \Pi_{h} \boldsymbol{u}^{n}\right|_{1}+\left|\Pi_{2 h} \Pi_{h} \boldsymbol{u}^{n}-\Pi_{2 h} \boldsymbol{U}_{h}^{n}\right|_{1} \\
\leq & C h^{2}\left\|\boldsymbol{u}^{n}\right\|_{3}+0+C\left\|\Pi_{h} \boldsymbol{u}^{n}-\boldsymbol{U}_{h}^{n}\right\|_{h} \leq C\left(h^{2}+\tau\right) .
\end{aligned}
$$

Similarly, the result for $\tau \sum_{i=1}^{n}\left\|p^{i}-J_{2 h} P_{h}^{i}\right\|_{0}^{2}$ can be derived by (3.42)-(3.44). The proof is complete. 


\section{Numerical Examples}

In this section, some numerical results are provided to confirm the theoretical analysis.

Example 4.1. The viscosity coefficient $\nu=1$. The boundary/inital conditions and the source term $\boldsymbol{f}$ are chosen according to the exact solutions

$$
\begin{aligned}
& u_{1}=e^{-t}\left(x_{1}^{2}-2 x_{1}^{3}+x_{1}^{2}\right)\left(4 x_{2}^{3}-6 x_{2}^{2}+2 x_{2}\right), \\
& u_{2}=-e^{-t}\left(x_{2}^{4}-2 x_{2}^{3}+x_{2}^{2}\right)\left(4 x_{1}^{3}-6 x_{1}^{2}+2 x_{1}\right), \\
& p=10 e^{-t}\left(2 x_{1}-1\right)\left(2 x_{2}-1\right) .
\end{aligned}
$$

The final time is set $T=1.0$ and the domain $\Omega=(0,1)^{2}$. A regular triangulation with $M+1$ nodes in both horizontal and vertical directions is made for the domain $\Omega$.

In order to demonstrate the error estimates in Theorems 3.1 and 3.2, we choose $\tau=O\left(h^{2}\right)$ and list the numerical results with respect to $t=0.1,0.6,1.0$ in Tables 4.1-4.3, respectively. It

Table 4.1: The numerical errors at $t=0.1$ of Example 4.1.

\begin{tabular}{|lllll|}
\hline$M \times M$ & $8 \times 8$ & $16 \times 16$ & $32 \times 32$ & $64 \times 64$ \\
\hline$\left\|\boldsymbol{u}^{n}-\boldsymbol{U}_{h}^{n}\right\|_{h}$ & $1.5917 \mathrm{e}-02$ & $8.0380 \mathrm{e}-03$ & $4.0274 \mathrm{e}-03$ & $2.0149 \mathrm{e}-03$ \\
Rate & $/$ & 0.98568 & 0.99700 & 0.99915 \\
$\left\|\Pi_{h} \boldsymbol{u}^{n}-\boldsymbol{U}_{h}^{n}\right\|_{h}$ & $2.7549 \mathrm{e}-03$ & $7.7676 \mathrm{e}-04$ & $1.8784 \mathrm{e}-04$ & $4.9497 \mathrm{e}-05$ \\
Rate & $/$ & 1.8264 & 2.0480 & 1.9241 \\
$\left\|\boldsymbol{u}^{n}-\Pi_{2 h} \boldsymbol{U}_{h}^{n}\right\|_{1}$ & $5.0596 \mathrm{e}-03$ & $1.2856 \mathrm{e}-03$ & $3.1505 \mathrm{e}-04$ & $8.0154 \mathrm{e}-05$ \\
Rate & $/$ & 1.9766 & 2.0288 & 1.9748 \\
$\left\|p^{n}-P_{h}^{n}\right\|_{0}$ & $5.4990 \mathrm{e}-01$ & $2.6876 \mathrm{e}-01$ & $1.3356 \mathrm{e}-01$ & $6.6682 \mathrm{e}-02$ \\
Rate & $/$ & 1.0329 & 1.0088 & 1.0021 \\
$\left\|J_{h} p^{n}-P_{h}^{n}\right\|_{0}$ & $1.4260 \mathrm{e}-01$ & $3.6042 \mathrm{e}-02$ & $8.9144 \mathrm{e}-03$ & $2.2528 \mathrm{e}-03$ \\
Rate & $/$ & 1.9842 & 2.0154 & 1.9844 \\
$\left\|p^{n}-J_{2 h} P_{h}^{n}\right\|_{0}$ & $1.8945 \mathrm{e}-01$ & $4.7656 \mathrm{e}-02$ & $1.1841 \mathrm{e}-02$ & $2.9784 \mathrm{e}-03$ \\
Rate & $/$ & 1.9911 & 2.0089 & 1.9911 \\
\hline
\end{tabular}

Table 4.2: The numerical errors at $t=0.6$ of Example 4.1 .

\begin{tabular}{|lllll|}
\hline$M \times M$ & $8 \times 8$ & $16 \times 16$ & $32 \times 32$ & $64 \times 64$ \\
\hline$\left\|\boldsymbol{u}^{n}-\boldsymbol{U}_{h}^{n}\right\|_{h}$ & $9.6578 \mathrm{e}-03$ & $4.8755 \mathrm{e}-03$ & $2.4427 \mathrm{e}-03$ & $1.2221 \mathrm{e}-03$ \\
Rate & $/$ & 0.98615 & 0.99705 & 0.99915 \\
$\left\|\Pi_{h} \boldsymbol{u}^{n}-\boldsymbol{U}_{h}^{n}\right\|_{h}$ & $1.7073 \mathrm{e}-03$ & $4.7445 \mathrm{e}-04$ & $1.1447 \mathrm{e}-04$ & $3.0144 \mathrm{e}-05$ \\
Rate & $/$ & 1.8474 & 2.0512 & 1.9250 \\
$\left\|\boldsymbol{u}^{n}-\Pi_{2 h} \boldsymbol{U}_{h}^{n}\right\|_{1}$ & $3.0916 \mathrm{e}-03$ & $7.8184 \mathrm{e}-04$ & $1.9142 \mathrm{e}-04$ & $4.8692 \mathrm{e}-05$ \\
Rate & $/$ & 1.9834 & 2.0302 & 1.9750 \\
$\left\|p^{n}-P_{h}^{n}\right\|_{0}$ & $3.3353 \mathrm{e}-01$ & $1.6301 \mathrm{e}-01$ & $8.1008 \mathrm{e}-02$ & $4.0444 \mathrm{e}-02$ \\
Rate & $/$ & 1.0329 & 1.0088 & 1.0021 \\
$\left\|J_{h} p^{n}-P_{h}^{n}\right\|_{0}$ & $8.6491 \mathrm{e}-02$ & $2.1860 \mathrm{e}-02$ & $5.4069 \mathrm{e}-03$ & $1.3664 \mathrm{e}-03$ \\
Rate & $/$ & 1.9842 & 2.0154 & 1.9844 \\
$\left\|p^{n}-J_{2 h} P_{h}^{n}\right\|_{0}$ & $1.1491 \mathrm{e}-01$ & $2.8905 \mathrm{e}-02$ & $7.1817 \mathrm{e}-03$ & $1.8065 \mathrm{e}-03$ \\
Rate & $/$ & 1.9911 & 2.0089 & 1.9911 \\
\hline
\end{tabular}


Table 4.3: The numerical errors at $t=1.0$ of Example 4.1.

\begin{tabular}{|lllll|}
\hline$M \times M$ & $8 \times 8$ & $16 \times 16$ & $32 \times 32$ & $64 \times 64$ \\
\hline$\left\|\boldsymbol{u}^{n}-\boldsymbol{U}_{h}^{n}\right\|_{h}$ & $6.4633 \mathrm{e}-03$ & $3.2659 \mathrm{e}-03$ & $1.6372 \mathrm{e}-03$ & $8.1915 \mathrm{e}-04$ \\
Rate & $/$ & 0.98477 & 0.99622 & 0.99906 \\
$\left\|\Pi_{h} \boldsymbol{u}^{n}-\boldsymbol{U}_{h}^{n}\right\|_{h}$ & $1.0250 \mathrm{e}-03$ & $2.7257 \mathrm{e}-04$ & $6.9173 \mathrm{e}-05$ & $1.7358 \mathrm{e}-05$ \\
Rate & $/$ & 1.9109 & 1.9784 & 1.9946 \\
$\left\|\boldsymbol{u}^{n}-\Pi_{2 h} \boldsymbol{U}_{h}^{n}\right\|_{1}$ & $1.9991 \mathrm{e}-03$ & $4.9681 \mathrm{e}-04$ & $1.2390 \mathrm{e}-04$ & $3.0953 \mathrm{e}-05$ \\
Rate & $/$ & 2.0086 & 2.0036 & 2.0010 \\
$\left\|p^{n}-P_{h}^{n}\right\|_{0}$ & $2.2345 \mathrm{e}-01$ & $1.0923 \mathrm{e}-01$ & $5.4300 \mathrm{e}-02$ & $2.7110 \mathrm{e}-02$ \\
Rate & $/$ & 1.0325 & 1.0084 & 1.0021 \\
$\left\|J_{h} p^{n}-P_{h}^{n}\right\|_{0}$ & $5.7482 \mathrm{e}-02$ & $1.4370 \mathrm{e}-02$ & $3.5926 \mathrm{e}-03$ & $8.9815 \mathrm{e}-04$ \\
Rate & $/$ & 2.0000 & 2.0000 & 2.0000 \\
$\left\|p^{n}-J_{2 h} P_{h}^{n}\right\|_{0}$ & $7.6642 \mathrm{e}-02$ & $1.9161 \mathrm{e}-02$ & $4.7901 \mathrm{e}-03$ & $1.1975 \mathrm{e}-03$ \\
Rate & $/$ & 2.0000 & 2.0000 & 2.0000 \\
\hline
\end{tabular}

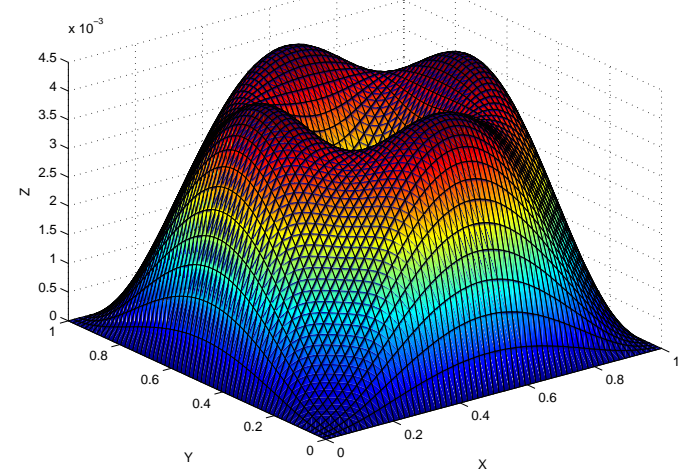

(a) $|\boldsymbol{u}|$.

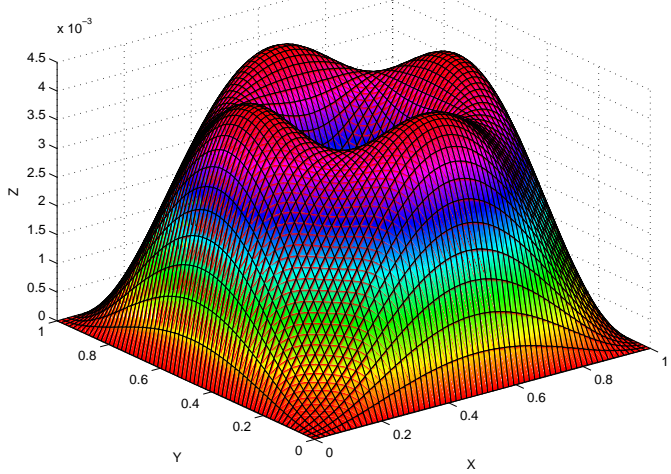

(b) $\left|\boldsymbol{U}_{h}\right|$.

Fig. 4.1. The graphics on mesh $64 \times 64$ at $t=1.0$ of Example 4.1.

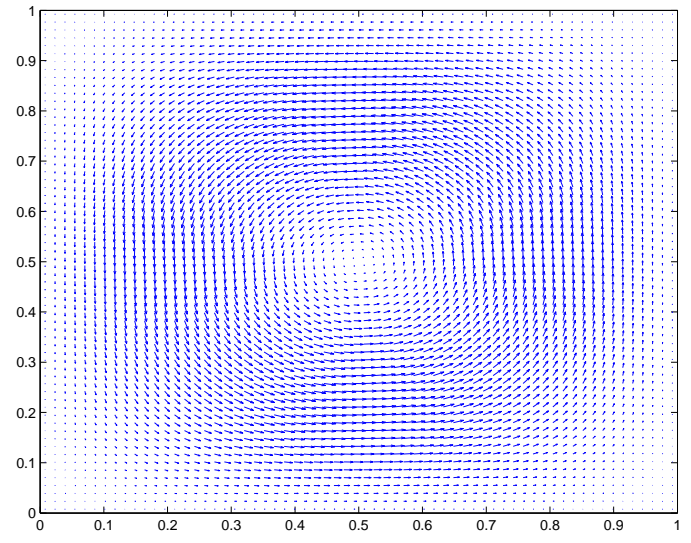

(a) The vector field of $\boldsymbol{u}^{n}$

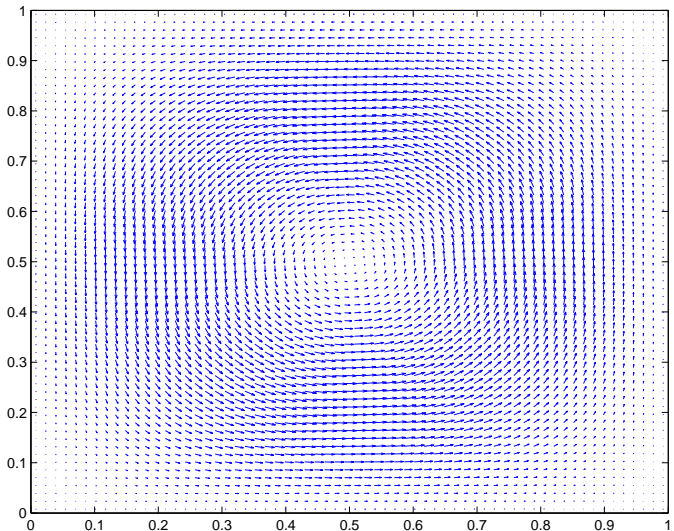

(b) The vector field of $\boldsymbol{U}_{h}^{n}$.

Fig. 4.2. The graphics on mesh $64 \times 64$ at $t=1.0$ of Example 4.1. 


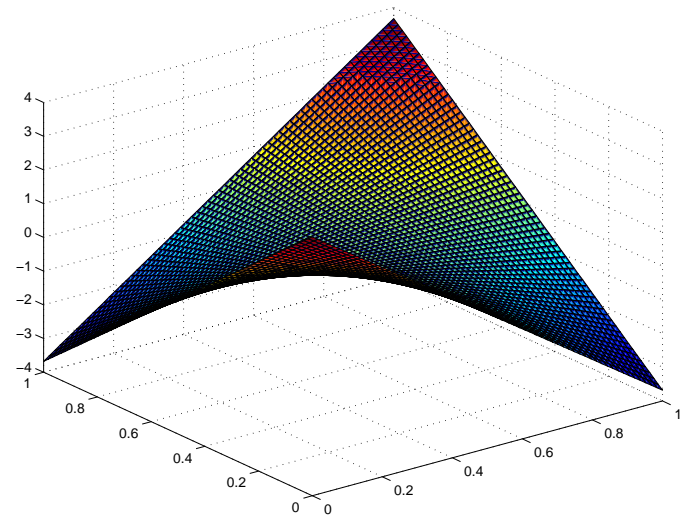

(a) The exact solution $p^{n}$

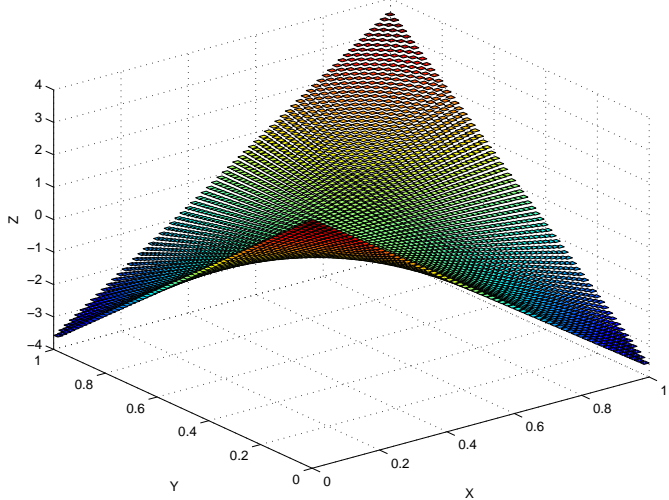

(b) The numerical solution $P_{h}^{n}$.

Fig. 4.3. The graphics on mesh $64 \times 64$ at $t=1.0$ of Example 4.1 .

can been see that the convergence rates of order $O\left(h^{2}\right)$ for the velocity $\boldsymbol{u}$ are in good agreement with the theoretical analysis. Moreover, the convergence rates are of order $O\left(h^{2}\right)$ for the pressure $p$ in $L^{\infty} \times L^{2}$-norm, although the theoretical analysis was given only in the $L^{2} \times L^{2}$-norm. At the same time, we also present the graphics of the exact and numerical solutions at $t=1.0$ on mesh $64 \times 64$ (see Figs. 4.1-4.3).

Example 4.2. This is a lid-driven cavity flow problem. The classical problem of the closed cavity driven by the motion of a leaky lid has been used rather extensively as a validation test case by many authors $[37,38]$. In this problem, a unit velocity is specified along the entire top surface and zero velocity on the other surfaces as shown in Fig. 4.4.

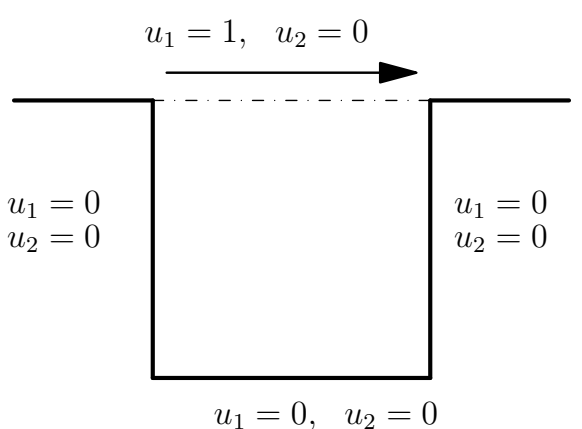

Fig. 4.4. Model description of Example 4.2.

We consider the flow for different Reynolds numbers on a fixed mesh with $h=1 / 64$. For low Reynolds number $(R e=1)$, the flow has only vortex located above the center. When the Reynolds number increases, the flow pattern starts to form reverse circulation cells in two lower corners (see Fig. 4.5), which shows that the results obtained herein are in good agreement with the phenomenon discussed in [38-40].

Acknowledgements. This work is supported by National Natural Science Foundation of China (Nos. 11671369; 11271340). 


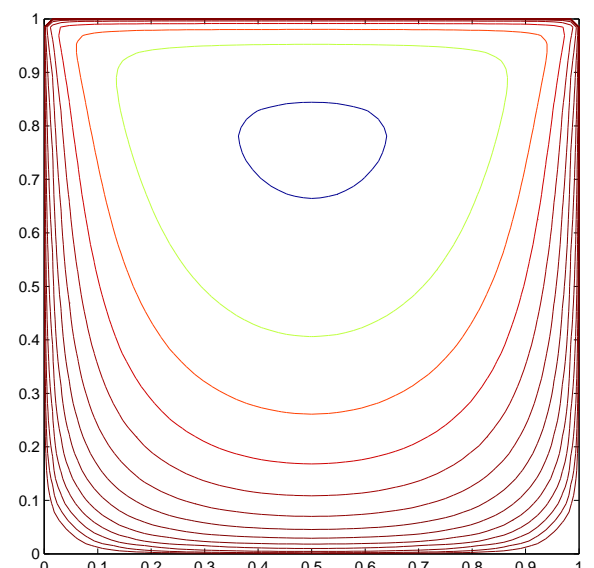

(a) $R e=1$

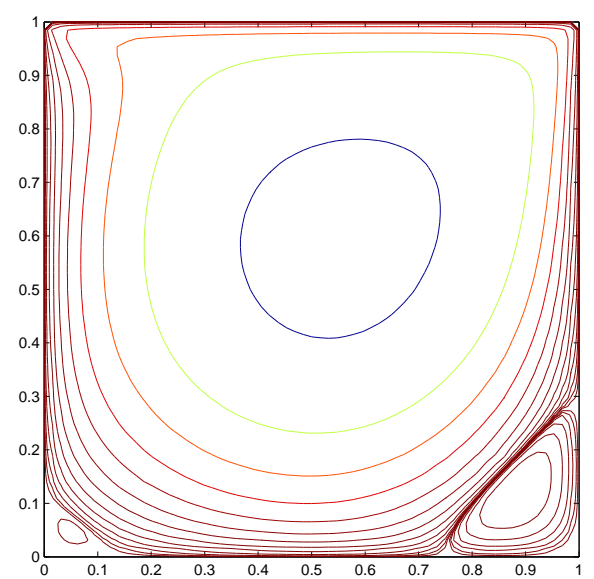

(c) $R e=500$.

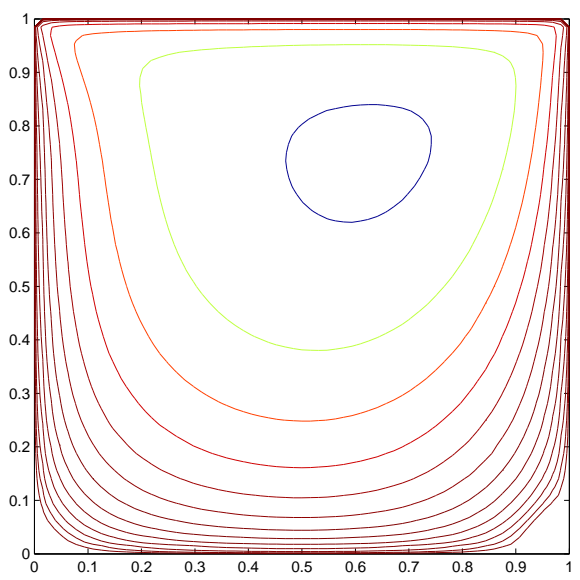

(b) $R e=100$.

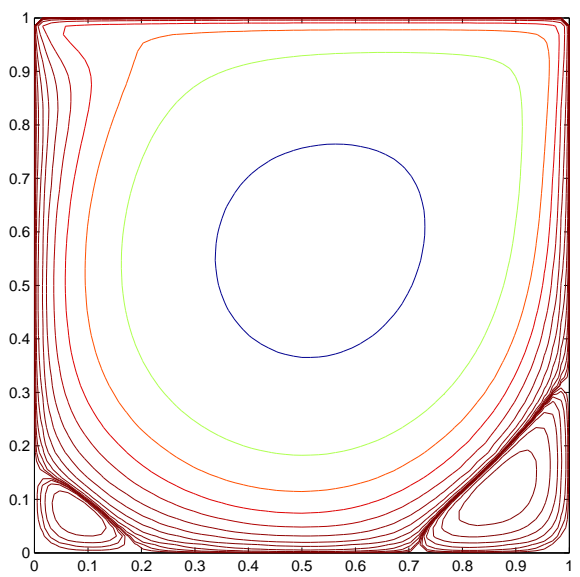

(d) $R e=1000$.

Fig. 4.5. Velocity fields of Example 4.2.

\section{References}

[1] V. Girault, P.A. Raviart, Finite element method for Naviet-Stokes equations: Theory and Algorithms, Springer-Verlag, Berlin, 1987.

[2] R. Teman, Navier-Stokes equations: Theory and Numerical analysis, North Holland, Amsterdam, New-York-Oxford, 1977.

[3] C. Wang, J.G. Liu, Analysis of finite difference schemes for unsteady Navier-Stokes equations in vorticity formulation, Numer. Math., 91 (2002), 543-576.

[4] Z.L. Li, C. Wang, A fast finite difference method for solving Navier-Stokes equations on irregular domains, Communications in Math. Sci., 1 (2003), 181-197.

[5] T.Y. Hou, B.T.R. Wetton, Convergence of a finite difference scheme for the Navier-Stokes equations using vorticity boundary conditions, SIAM J. Numer. Anal., 29 (1992), 615-639.

[6] J.C. Strikwerda, Finite difference methods for the Stokes and Navier-Stokes equations, SIAM J. Sci. Stat. Comput., 5 (1984), 56-68.

[7] Y. He, Optimal error estimate of the penalty finite element method for the time-dependent NavierStokes equations, Math. Comput., 74 (2005), 1201-1216.

[8] Y. He, A fully discrete stablized finite element method for the time-dependent Navier-Stokes 
problem, IMA J. Numer. Anal., 23 (2003), 665-691.

[9] Y. He, J. Li, A stablized finite element method based on local polynomial pressure projection for the stationary Navier-Stokes equations, Appl. Numer. Math., 58 (2008), 1503-1514.

[10] Y. He, A two-level finite element Galerkin method for the nonstationary Navier-Stokes equations I: Spatial Discretization, J. Comput. Math., 22 (2004), 21-32.

[11] Y. He, H. Miao, C. Ren, A two-level finite element Galerkin method for the nonstationary NavierStokes equations II: Time Discretization, J. Comput. Math., 22 (2004), 33-54.

[12] J.G. Heywood, R. Rannacher, Finite Element Approximation of the Nonstationary Navier-Stokes Problem. I. Regularity of Solutions and Second-Order Error Estimates for Spatial Discretization, SIAM J. Numer. Anal., 19 (1982) 275-311.

[13] J.G. Heywood, R. Rannacher, Finite element approximation of the nonstationary Navier-Stokes problem. II. Stability of solutions and error estimates uniform in time, SIAM J. Numer. Anal., 23 (1986), 750-777.

[14] J.G. Heywood And R. Rannacher, Finite Element Approximation of the Nonstationary NavierStokes Problem, Part III. Smoothing Property and Higher Order Error Estimates for Spatial Discretization, SIAM J. Numer. Anal., 25 (1988), 489-512.

[15] J.G. Heywood And R. Rannacher, Finite-element approximation of the nonstationary NavierStokes problem. IV. Error analysis for second-order time discretization, SIAM J. Numer. Anal., 27 (1990), 353-384.

[16] H. Han, A finite element approximation of Navier-Stokes equations using nonconforming elements, J. Comput. Math., 2 (1984), 77-88.

[17] X. Xu, On the accuracy of nonconforming quadrilateral $Q_{1}$ element approximation for the NavierStokes problem, SIAMJ. Numer. Anal., 38 (2000), 17-39.

[18] Y. He, Two-level method based on finite element and Crank-Nicolson extrapolation for the timedependent Navier-Stokes equations, SIAM J. Numer. Anal., 41:4 (2003), 1263-1285.

[19] Y. He, W. Sun, Stability and convergence of the Crank-Nicolson/Adams-Bashforth scheme for the time-dependent Navier-Stokes equations, SIAM J. Numer. Anal., 45:2 (2007), 837-869.

[20] Y. He, The Euler implicit/explicit scheme for the 2D time-dependent Navier-Stokes equations with smooth or non-smooth initial data, Math. Comp., 77:264 (2008), 2097-2124.

[21] Y. He, K. Liu, A multilevel finite element method in space-time for the Navier-Stokes problem, Numer. Methods Partial Differential Equations, 21:6 (2005), 1052-1078.

[22] D. Shi, J. Ren, X. Hao, A new second order nonconforming mixed finite element scheme for the stationary Stokes and Navier-Stokes equations, Appl. Math. Comput., 207 (2009), 462-477.

[23] D. Shi, W. Gong, J. Ren, A new stable second nonconforming mixed finite element scheme for the stationary Stokes and Navier-Stokes equations, Math. Comput. Modell., 53 (2011), 1956-1969.

[24] D. Shi, J. Ren, Nonconforming mixed finite element approximation to the stationary Navier-Stokes equations on anistropic meshes, Nonl. Anal. TMA, 71 (2009), 3842-3852.

[25] Y. Achdou, J.L. Guermond, Convergence analysisi of a finite element/Lagrange-Galerkin method for the incompressible Navier-Stokes equations, SIAM J. Numer. Anal., 37 (2000), 799-826.

[26] R. Bermejo, P. Galán del Sastre, A second order in time modufied Lagrange-Galerkin finite element method for the incompressible Navier-Stokes equations, SIAM J. Numer. Anal., 50 (2012), 30843109.

[27] Z. Si, J. Wang, W. Sun, Unconditional stability and error estimates of modified characteristics FEMs for the Navier-Stokes equations, Numer. Math., 134 (2016), 139-161.

[28] P. Chandrashekar, Discontinuous Galerkin method for Naviet-Stokes equations using kinetic vector splitting, J. Comput. Phys., 233 (2013), 527-551.

[29] N. Fehn, W.A. Wall, M. Kronbichler, On the stability of projection methods for the incompressible Navier-Stokes equations based on high-order discontinuous Galerki discretizations, J. Comput. Phys., 351 (2017), 392-421.

[30] J. Ren, Y. Ma, A superconvergent nonconforming mixed finite element method for the Navier- 
Stokes equations, Numer. Methods Partial Differential Equations, 32 (2016), 646-660.

[31] C. Xu, D. Shi, X. Liao, Low order nonconforming mixed finite element method for nonstationary incompressible Navier-Stokes equations, Appl. Math. Mech. -Engl. Ed., 37 (2016), 1095-1112.

[32] J. Hun, Z. Shi, Constrained quadrilateral nonconforming rotated $\mathcal{Q}_{1}$ element, J. Comput. Math., 23:5 (2005), 561-585.

[33] H. Liu, N. Yan, Superconvergence analysis of the nonconforming quadrilateral linear-constant scheme for Stokes equations, Adv. Comput. Math., 29 (2008), 375-392.

[34] R.A. Adams, J.J.F. Fournier, Sobolev spaces, Academic press, 2003.

[35] R. Rannacher, S. Turek, Simple nonconforming quadrilateral Stokes element, Numer. Methods Partial Equations, 8 (1992), 97-111.

[36] C. Park, D. Sheen, $P_{1}$ nonconforming quadrilateral finite element methods for second-order elliptic problem, SIAM J. Numer. Anal., 41 (2003), 624-640.

[37] M. Li, D. Shi, Y. Dai, Stabilized low order finite elements for Stokes equations with damping, J. Math. Anal. Appl., 435 (2016), 646-660.

[38] U. Ghia, K. Ghia, C. Shin, High-Re solutions for incompressible flows using the Navier-Stokes equations and a multigrid method, J. Comput. Phys., 48 (1982), 387-411.

[39] S. Kaya, B. Riviere, A two-grid stabilization method for solving the steady-state Navier-Stokes equations, Numer. Methods Partial Differential Equations, 22 (2006), 728-743.

[40] H. Han, M. Yan, A mixed finite element method on a staggred mesh for Navier-Stokes equations, J. Comput. Math., 26 (2008), 816-824. 